\title{
Implementation of Cognitive Radio Networks to evaluate spectrum management strategies in real-time
}

\author{
Alessandro Raschellà*, Anna Umbert \\ Department of Signal Theory and Communications, Universitat Politècnica de Catalunya (UPC), c/ Jordi Girona,1-3, Campus Nord, Edificio D4, \\ Barcelona 08034, Spain
}

\section{A R T I C L E I N F O}

\section{Article history:}

Received 8 May 2015

Revised 5 November 2015

Accepted 8 November 2015

Available online $\mathrm{xxx}$

\section{Keywords:}

Cognitive radio

Opportunistic network

Spectrum management

Testbed

\begin{abstract}
A B S T R A C T
This paper illustrates a Universal Software Radio Peripheral (USRP)-based real-time testbed that is able to evaluate different spectrum management solutions that exploit the Cognitive Radio (CR) paradigm. The main objective of this testbed is to provide an accurate and realistic platform by which the performance of innovative spectrum management solutions for a wide set of scenarios and use cases in the context of Opportunistic Networks (ONs) and Cognitive Radio Networks (CRNs) can be entirely validated and assessed before their implementation in real systems. Real-time platforms are essential to carry out significant studies and to accurately assess the performance of innovative solutions before their implementation in the real world. This work provides a comprehensive description of the testbed, highlighting many interesting implementation details and illustrating its applicability for different studies that rely on the CR paradigm. Then, a particular application in a realistic Digital Home (DH) scenario is also illustrated, which allows demonstrating the effectiveness of the real-time testbed and assessing its practicality in terms of user-perceived end-to-end Quality of Experience $(\mathrm{QoE})$ in a realistic environment.
\end{abstract}

(C) 2015 Elsevier B.V. All rights reserved.

\section{Introduction}

The Cognitive Radio (CR) paradigm has been considered as an innovative solution to mitigate the spectrum scarcity problem by enabling Dynamic Spectrum Access (DSA), designed to conciliate the existing conflicts between the ever-increasing spectrum demand growth and the currently inefficient spectrum utilization $[1,2]$. The basic idea of DSA is to provide proper solutions that allow sharing the radio spectrum among several radio communication systems for the sake of optimizing the overall spectrum utilization.

With the advent of CR as a key enabler of DSA, several papers proclaimed the need for Cognitive Radio Networks (CRNs), which allow a wireless communication system based on the so-called cognitive cycle that enables observing the environment, acting and learning to optimize its performance. For instance, in [3,4], a CRN is defined as a wireless network with the capabilities of radio environment awareness, autonomous decision-making, adaptive reconfiguration of its infrastructure and intelligent learning from experience of a continuously changing environment to solve the challenges of efficient spectrum management and high-quality end-to-end performance.

\footnotetext{
* Corresponding author. Tel./fax: +34 934017195.

E-mail address: ale.raschella@gmail.com, alessandror@tsc.upc.edu, annau@tsc.upc.edu (A. Raschellà).
}

Furthermore, several papers have illustrated the expected benefits of developing cognitive management functionalities, which support CRNs in exploiting the mentioned CR capabilities in many specific scenarios [3-5]. For instance, in [5], a cognitive management framework is illustrated to carry out an autonomous optimization of resource usage in next-generation home networks. The proposed framework is able to autonomously improve the performance of network nodes in a dynamic environment according to the aims, restrictions and policy regulations formulated by network stakeholders. In [6], cognitive management functionalities are proposed to improve the efficiency of medical applications, and a novel cognitive architecture defined as Co-health is designed to exploit available knowledge and previous experience to support electronic healthcare, especially in emergency situations. In [7], the authors propose considering cognitive management systems for provisioning efficient applications in the Future Internet (FI). The main objective of the FI is to provide new and emerging applications through a wide range of Internet-enabled devices. Specifically, the solution proposed in [7] is based on the Opportunistic Networks (ONs) defined as extended infrastructures, temporarily created to serve specific regions providing application needs, which follow the policies dictated by the operator. Because of the temporary nature of the ONs and the highly dynamic nature of the environment, including traffic and application issues, as well as the need to identify radio resource opportunities, solutions incorporating the autonomous decision-making and reconfigurability 
mechanisms provided by a cognitive management system are deemed essential.

On the other hand, several research works have promoted the usage of specific analytical tools to assist in cognitive management functionalities for spectrum management by exploiting different forms of cognition of the radio environment. Some examples of the most popular criteria of cognition considered in the literature are reinforcement learning [8,9], Partially Observable Markov Decision Processes (POMDPs) [10-12], fuzzy logic tools [13] and game theory solutions $[14,15]$.

These proposals have been demonstrated to efficiently support spectrum management in different scenarios and use cases. However, the introduction of advanced cognitive management functionalities relying on some form of cognitive support to assist the spectrum management decision-making process has also arisen in the literature. This is the case for instance of the Functional Architecture (FA) proposed in [16] by the European Telecommunications Standardization Institute (ETSI) for Reconfigurable Radio Systems (RRSs) and its later extension for ONs [17]. In this context, in [18], a generic cognitive management FA for assisting the spectrum management decisionmaking process has been proposed based on the fittingness factor concept defined in [19]. In detail, this architecture integrates the fittingness factor to track the suitability of spectral resources to support a set of heterogeneous services subject to unknown changes in interference conditions.

Spectrum management policies supported by cognitive management functionalities proposed in the literature frequently rely on analytical models and off-line system-level simulators. The employ of such tools is typical within the research and industrial communities and can be convenient for achieving preliminary performance results. However, to carry out appropriate and relevant studies and to properly evaluate the performance of innovative solutions before progressing to a prototype or full-scale deployment, assessment on realistic platforms is crucial as a step towards implementation in real systems. In this context, real-time platforms enable the emulation of realistic scenarios to test algorithms, applications, protocols and policies under realistic conditions and represent a powerful tool for assessing the Quality of Experience (QoE) of end-users that could not be obtained through off-line simulations, as well as the Quality of Service (QoS).

Guided by this motivation, this paper provides a comprehensive description of a real-time testbed based on reconfigurable Universal Software Radio Peripheral (USRP) devices that can transmit/receive in different dynamically configured frequencies. This testbed has been envisaged, implemented and validated for the evaluation of a cognitive management functional architecture inspired from [16-18] in a real environment. In detail, this solution provides a framework to assess innovative spectrum management strategies exploiting the CR paradigm that have applicability in a wide set of scenarios and use cases. The functionalities of this architecture have already allowed achieving satisfactory results as published in referenced papers [2023]; notwithstanding, these publications either provide a general overview or address particular testbed issues and capabilities, and they do not provide an overall or exhaustive vision of the presented platform. Then, the aim of this work is twofold: (i) to provide a very detailed description of the whole developed platform, its entire potential and its applicability, with a holistic vision and discussion of the testbed, and demonstrate how it can be useful in a wide range of studies and scenarios; and (ii) to illustrate a particular application in a realistic Digital Home (DH) scenario to assess the practicability of the platform in terms of user-perceived end-to-end QoE, also in a realistic environment.

The rest of the paper is organized as follows. Section 2 provides a review of previous related works. On the one hand, the analysis of the state of the art in terms of spectrum management solutions in CRNs is presented; on the other hand, this section illustrates several
CR platforms found in the literature and describes the motivation for carrying out the new testbed presented in this paper. Section 3 discusses the applicability of the testbed, providing a general description of the previous works from authors whose results have been achieved through the platform in a broad set of scenarios and use cases. Then, Section 4 provides a holistic overview of the testbed architecture, including details about the implementation, the designed functionalities and their applicability. In Section 5, a detailed DH entertainment application carried out through the testbed is illustrated to assess the capabilities of the emulation platform also in a realistic scenario. Finally, Section 6 provides concluding remarks.

\section{Related works and motivations}

Spectrum management solutions in CRNs are responsible for proper utilization of the radio resources, and they are decisive to allow spectrum sharing among different radio systems, guaranteeing QoS requirements for different service classes without causing any interference. Hence, spectrum management functionalities are an interesting topic of research for different studies in the context of CRNs. For instance, in [24], the authors analyse different wideband spectrum sensing policies for CRNs, discussing the advantages and disadvantages of each solution. The research work developed in [25] proposes a game theoretic framework for joint spectrum sensing and spectrum access by considering the mutual influence between sensing and access for unlicensed users or Secondary Users (SUs) in two different scenarios: a synchronous scenario where the primary network is slotted, and an asynchronous scenario characterized by a non-slotted network. In [26], a cooperative channel state learning method based on a Bayesian learning rule for multi-channel sensing in different scenarios of the SUs is proposed. In [27], the authors focus their attention on a hierarchical DSA model to open the licensed spectrum to SUs while limiting the interference perceived by licensed ones or Primary Users (PUs). In particular, the basic components of this work include spectrum opportunity identification, spectrum opportunity exploitation, and a regulatory policy. The studies carried out in [28] and [29] propose strategies where SUs listen to feedback parameters that allow achieving information on the licensed spectrum channels. In detail, [28] focuses on an opportunistic spectrum sharing scheme where SUs can co-exist with the PUs; precisely, they can achieve feedback of licensed channel quality information that allows defining transmission parameters, such as the optimal transmit power and the transmission rate. Then, SUs can consider such information to enable the maximization of their own throughput. James et al. [29] propose a transmission scheme for SUs co-existing with a primary system based on the Automatic Repeat reQuest (ARQ) policy. Based on the ACK (positive acknowledge) or the NACK (negative acknowledge) message from the PU, this scheme exploits several probing time slots to achieve a general overview on the primary channel condition and operate accordingly with suitable transmission modes. In [30], the authors introduce a DSA algorithm for the SUs that do not know the interference behaviour of the PUs. Then, this paper analyses the SUs' behaviour in the licensed channel, demonstrating that it represents a renewal process. Finally, through the Renewal Theory [31], the authors perform an in-depth study of the interference provoked by the SUs.

Several research works rely on POMDPs that combine partial observations of the radio environment at specific periods of time with a statistical characterization of the system dynamics. In this direction, [10] proposes opportunistic spectrum access approaches to channels that can be either busy or idle, assuming a single SU. In [11,12], the use of a POMDP for spectrum selection in CRNs is proposed; moreover, these studies do not rely only on binary (i.e., idle/busy) measurements but instead consider a generalization in which the temporal variation of each available channel is able to capture different degrees of interference. Furthermore, different decision-making 
criteria for spectrum selection that can be found in the literature rely on databases that record historical information about the occupation of the different channels [32,33]. This type of information can be used to build predictive models on spectrum availability, as in [33]. In [34], an adaptive spectrum decision framework is presented, taking into account different types of applications, while in [35], a radio resource management method using both long- and short-term historical information is analysed.

In [36], the authors firstly note the necessity of strengthening noncooperative solutions in the context of the CR in terms of energy efficiency; then, they provide an in-depth study of energy-efficient spectrum sensing, spectrum sharing and deployment of CRN strategies from, respectively, micro, meso and macro perspectives. In [18], an FA for assisting the spectrum management decision-making process is proposed. In detail, it implements a framework that allows managing the most relevant knowledge stored in a specific database characterizing the radio environment and then assisting the spectrum management decision-making process accordingly.

From the analysis of the state of the art, it clearly emerges that there exists an important number of active research studies on spectrum management for CRNs, including spectrum sensing and spectrum sharing from the physical layer to the network one, with the aim of strengthening the use of the precious radio resources. Motivated by this consideration, the testbed presented in this paper has been designed, implemented and validated to provide a real-time platform on which to develop and assess innovative solutions for spectrum management in CRNs in a realistic environment. The versatile tool described in this work is a suitable and powerful platform for emulating spectrum sensing, spectrum opportunity identification and decisionmaking spectrum selection solutions in a broad variety of scenarios and use cases.

However, in the domain of CR research, several innovative emulation tools and real-time testbeds have already been proposed in the past to provide spectrum management solutions. For instance, in [36], the authors considered a testbed based on IEEE 802.15.4/ZigBee radios to implement an experiment demonstrating the power consumption of spectrum-sensing solutions based on energy detection. Then, they analysed the importance of appropriately developing spectrum-sensing solutions to reduce this power consumption.

In [37], the authors propose an experimental testbed based on the Berkeley Emulation Engine 2 (BEE2) platform and a multi-Field Programmable Gate Array (FPGA) emulation engine. The BEE2 can connect up to 18 radio front-ends, which can be configured as PUs or SUs. The authors illustrate how it is possible to experiment different sensing policies and design several metrics and use cases, which enable the assessment of such sensing functionalities through this testbed.

Another valuable example of a real-time platform is the Iris software architecture, which has been specifically developed for building highly reconfigurable radio network testbeds based on intelligent observations that the radio makes about its surroundings [38]. Its primary research application is to enable a wide range of DSA and CR experiments. It is a General Purpose Processor (GPP)-based radio architecture and uses Extensible Markup Language (XML) documents to describe the radio structure. Iris includes many of the features required by several emerging CR standards, such as IEEE 802.22, which addresses the use of Very High Frequencies (VHFs) and Ultra High Frequencies (UHFs) TV band spectrum through a non-interfering basis to provide Wireless Regional Area Networks (WRANs) in areas of low population density, such as in rural areas.

The work proposed in [39] provides details of a distributed genetic algorithm based on a CR engine model for disaster communications and its development through a CR testbed relying on programmable radios. This platform exploits a Cognitive System Monitor (CSM) module, which allows adaptation of a programmable radio by classifying the observed channel and matching channel behaviour with operational goals. This module then sends these goals to a Wireless System Genetic Algorithm (WSGA) adaptive controller entity to evolve and optimize the radio operation. WSGA is then able to adjust several parameters, such as power, modulation and coding, to achieve the goals provided by the CSM.

The Virginia Tech CR Network (VT-CORNET) [40] is an open and highly reconfigurable testbed that allows the evaluation of independently developed CR engines, sensing techniques, applications, protocols, performance metrics, and algorithms in a real-world wireless environment. The current and planned testbed capabilities include 48 USRP2-based nodes spread over four floors of a building and equipped with a custom-made daughterboard spanning the frequency range from $100 \mathrm{MHz}$ to $4 \mathrm{GHz}$.

The Open Access Research Testbed (ORBIT) project provides another flexible real-time wireless platform [41]. This project was started with the objective of developing a large-scale open-access wireless networking testbed available to the research community working on next-generation protocols, middleware and applications. The project was then extended to support operations and several key technical upgrades, including the introduction of Software Defined Radio (SDR) capabilities and CR networking experiments through 28 boards, including USRP and USRP2.

All the above-mentioned valid platforms, as well as the testbed presented in this paper, provide the following functionalities, which are essential in the implementation of CR networking solutions: (i) cognitive capability, i.e., the ability to capture information from the radio environment; (ii) and reconfigurability, which enables the receiver and the transmitter parameters to be dynamically programmed and modified according to the radio environment. Notwithstanding, the real-time platform developed to provide spectrum management solutions and illustrated in this paper has been designed with specific aims that would not have been achieved with the features of the above-mentioned platforms. In fact, all the solutions implemented through the testbed have been developed and implemented to allow a reliable statistical characterization of the radio environment and consequently an efficient utilization of the available spectrum resources. In detail, this testbed offers further tools that enable the strengthening of the cognitive capability by statistical patterns that characterize the activity of the different devices sharing the spectrum and improvement of the CRN reactivity to changes by additional learning and adaptability capabilities. To this aim, the proposed FA implemented through the testbed introduces a standalone knowledge management domain to store in a database and manage the most relevant knowledge regarding the realistic radio environment out of the decision-making domain. This domain separation enables the development of generic cognitive management functionalities independently from the decision-making process. Knowledge management functionalities that exploit real-time information achieved through awareness processes and stored in apposite databases and that support the decision-making can find applicability in a wide set of scenarios and use cases as demonstrated in the referred papers [20-23]. Moreover, they provide satisfactory performance results in terms of QoS, QoE and reduction of the signalling, as will be widely explained throughout the next sections.

\section{Available configurations}

The testbed presented in this paper is a suitable platform for exploiting cognitive management functionalities in the context of spectrum management for many studies and use cases. In particular, the platform models a comprehensive CRN that can be easily configured to reflect a broad range of scenarios, providing researchers with a powerful tool for validating and optimizing their designs and algorithms before their implementation in real systems. In the following, different networks relying on the CR paradigm that have been envisaged and assessed through the testbed created by the authors are provided. 


\subsection{Opportunistic Networks}

The platform has been firstly considered to implement realistic ONs where innovative spectrum management solutions can be assessed and validated. ONs are defined as extended infrastructures, temporarily created to serve specific regions and providing application needs under certain circumstances. The lifecycle of an ON comprises the following phases [42]: (1) suitability determination, where the convenience of setting up a new $\mathrm{ON}$ is assessed according to the triggering situation, previous knowledge, policies, profiles, etc.; (2) creation, which includes the selection of the optimal, feasible configuration for the new ON (selection of the participant nodes, the spectrum and the routing pattern); (3) maintenance, which involves monitoring and controlling the QoS of the data flows involved in the $\mathrm{ON}$ and performing the appropriate corrective actions when needed; and (4) termination, when the motivations for the creation of the ON disappear or the ON can no longer provide the required QoS and, therefore, mechanisms should be provided to handle handovers and to keep applications alive if possible.

A common technical challenge in the ONs is proper management of the spectrum to be used for the transmission of data and control flows in any communication link in accordance with the requirements for this link depending on the applications to be supported. The spectrum management process can be divided into two differentiated steps. First, the spectrum opportunity identification is in charge of finding the set of possible frequency bands that are available for the link. Second, and based on the results of the previous step, the spectrum selection decides the most adequate band for the communication. Based on the FA proposed in [16], an extension was proposed in [17] to address ON management by considering decisive entities for the management of the spectrum resources, as briefly described in the following.

The Dynamic Spectrum Management (DSM) entity provides management of the spectrum availability conditions and related constraints to guide the spectrum selection decision-making.

The Cognitive management Systems for Coordinating the Infrastructure (CSCI) is mainly responsible for the activities before an $\mathrm{ON}$ is created. It is the functional entity in charge of acquiring and processing the context to carry out the ON suitability determination phase that detects the situations where an ON may be useful and determines whether the right conditions are in place for creating an ON. The CSCI delegates the actual creation, maintenance and termination of a given $\mathrm{ON}$ to the associated Cognitive systems for Managing the Opportunistic Network (CMON) functional entity.

The CMON controls the lifecycle of the ON from creation to termination. This includes the execution of the creation procedures to enforce the design obtained from the $\mathrm{CSCI}$, such as the assignment of the spectrum resources, the supervision of the ON during the maintenance phase, and the termination procedures.

The testbed illustrated in this paper provides a real-time platform that allows implementing a particularization of the FA proposed in [17] for ON creation and ON maintenance in a centralized manner $[20,21]$. This FA is illustrated in Fig. 1, and it enables emulation of the DSM, which provides the radio resource availability conditions through the spectrum opportunity identification procedure to guide the spectrum selection decision-making implemented in the CMON during the $\mathrm{ON}$ creation and $\mathrm{ON}$ maintenance phases. From the figure, it can be noticed that the decision-making entity in charge of the spectrum selection execution implemented in the CMON is supported by the following blocks: (i) the context awareness that provides constant feedback of the ON's experienced QoS to trigger reconfiguration or termination procedures in case of either a detriment of QOS or the loss of the resources; (ii) the control block that addresses the triggers of the execution of ON establishment, its reconfiguration and its termination; and (iii) the knowledge management block that interacts with the decision-making to enable better decisions in the

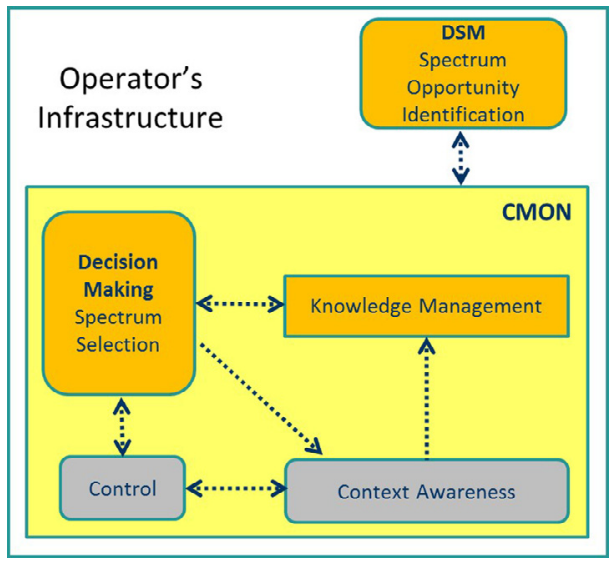

Fig. 1. FA for ONs implemented through the testbed.

future according to the statistical results learned from the radio environment.

These solutions have applicability in the possible scenarios and use cases defined in [43], such as: (1) "opportunistic coverage extension," which describes a situation in which a device cannot connect to the operator's infrastructure due to lack of coverage or a mismatch in the radio access technologies; (2) "opportunistic capacity extension," which depicts a situation in which a device cannot access the operator infrastructure due to the congestion of the available resources at the serving access node; and (3) "infrastructure supported opportunistic ad-hoc networking," which considers the creation of a localized, infrastructureless ON among several devices for a specific purpose (peer-to-peer communications, home networking, locationbased services, etc.).

The most relevant contribution of $[20,21]$ is the implementation through the testbed of a centralized framework based on the FA proposed in [17], providing new solutions for spectrum opportunity identification and spectrum selection and their validation in a realistic environment. The results have firstly validated the implementation conducted at the laboratory, providing an in-depth analysis of the reconfigurability capabilities of the ON links under varying interference conditions; then, the performance of the spectrum management solutions have been assessed experimentally. Moreover, a real-time demonstration of these functionalities has been performed and assessed in the context of the European project named Opportunistic Networks and Cognitive Management Systems for Efficient Application Provision in the Future Internet (OneFIT) [44]. In particular, it has been demonstrated in real-time how the platform allows the ON maintenance stage, monitoring and guaranteeing the quality in the communication in the Industrial, Scientific and Medical (ISM) spectrum band.

\subsection{Cognitive Radio Networks}

The platform has then been considered to emulate more general CRNs where efficient spectrum management solutions that relay on the cognitive cycle can be assessed. In detail, the cognitive cycle involves observations of the environment, analysis of these observations, decision-making to intelligently configure certain radio parameters and finally execution of the decisions via actions [1]. Analysis and decision can be supported by learning mechanisms that exploit the knowledge obtained from the execution of prior decisions, while the observation stage typically involves making measurements at several nodes of a CRN. These measurements must be reported to the entity in charge of analysing them to extract the relevant information about the radio environment and to execute the decisionmaking. Given that the observation stage can be costly in terms of 

practical requirements, such as signalling overhead and battery consumption, decision-making strategies able to efficiently operate with a minimum number of measurements are of high interest for enhancing CR operation.

In this context, the authors in $[22,23,45]$ proposed a spectrum selection decision-making framework based on the so-called belief vector to predict the environment dynamics, which allows avoiding measurements when possible. In detail, the belief vector assesses the probability that the radio environment is under specific conditions at a certain time based on past measurements (observations). Moreover, the proposed framework can be particularized to different observation strategies to determine the instants when measurements of the radio environment need to be performed, assessing the tradeoff existing between performance and observation requirements of the cognitive cycle. Some illustrative use cases where this framework can be applicable are: (1) a DH scenario in which different devices need to communicate, (2) a set of cognitive small cells deployed in a cellular network that make use of an additional spectrum to increase the network capacity, and (3) an opportunistic DeviceTo-Device (D2D) radio link created to extend the coverage of certain cellular terminals that are outside the coverage area of the cellular infrastructure.

The system model implemented in this testbed considers a set of radio links, each one intended to support data transmission between either a pair of terminals or between a terminal and an infrastructure node. The potential spectrum to be assigned to the different radio links is organized in a set of spectrum blocks (SBs) characterized by a central frequency and bandwidth. The available bit rate for the links in the SBs depends on both the propagation conditions between the link transmitter and receiver and the interference experienced at the receiver. Then, the considered problem consists of performing an efficient allocation of the SBs to the radio links by properly matching the bit rate requirements with the achievable bit rate in each SB. For that purpose, it is assumed that the different radio links are controlled by a centralized decision-making entity in charge of deciding the spectrum to be used by each radio link.

The interference evolution for the $i$ th SB is modelled as an ergodic discrete-time Markov process with the state transition probability from being in state $k$ at time $t$ and moving to state $k^{\prime}$ in the next time step $t+1$. It is assumed that the state of the $i$ th $\operatorname{SB} S^{(i)}(t)$ evolves independently from the other SBs and that the state evolution is independent from the assignments made by the spectrum selection policy. Each radio link with a data session in progress (referred to as an active link) will obtain a reward that measures the obtained performance depending on the interference state of the allocated SB at each time. Supposing the use of the $j$ th link, $r_{j, k}^{(i)}$ denotes the reward that this link receives when using its allocated SB $i$ and the interference state is $S^{(i)}(t)=k$. The reward is a metric between 0 and 1 capturing how suitable the $i$ th SB is for the $j$ th radio link, depending on the bit rate that can be achieved in this SB with respect to the bit rate required by the application. The spectrum selection policy proposed in $[22,23]$ and [45], executed at time $t$ for the $j$ th radio link, targets the maximization of the expected reward that the session will experience along its duration $D_{j}$.

The selection is made among the available SBs, i.e., those that are not allocated to any other radio link at the decision-making time $t$. The analysis of the future evolution of the reward in each of the SBs until the session ends exploits the measurements of the interference state of the different SBs carried out at specific time instants in the past, together with the statistical characterization of the interference dynamics in each SB. In detail, the statistical characterization of the interference dynamics in the $i$ th SB is given by the belief vector $\mathbf{b}^{(\mathbf{i})}(t)$.

The computation of the belief vector of the $i$ th SB at a certain time instant $t$ is performed recursively, starting from the last observation of the actual interference state that was taken in the $i$ th SB at time step $t-m^{(i)}$. In particular, the belief vector at a time instant $t>t-m^{(i)}$ can be obtained from the belief vector at the previous $m^{(i)}$ time steps using the state transition probabilities of the interference states recursively. Further details about the computation of the belief vector and the decision-making spectrum selection policy will be provided in Section 4.3.

Several observation strategies that specify the time instants when the actual interference state in each SB is measured have been developed. The observation strategy should make sure that the time elapsed between the last observation and the spectrum selection decision-making time $t$ is adequate enough to compute the belief vector and make accurate decisions. Moreover, because the observation stage can be very costly in terms of practical requirements, an observation strategy should guarantee an appropriate trade-off between performance and the number of measurements achieved through signalling procedures. Hence, papers $[22,23,45]$ consider the following three observation strategies:

(a) Instantaneous Measurements (IM) strategy: It consists of performing instantaneous measurements of the interference states in all the SBs at the time $t$ when a new session has to be established, i.e., at the time when the spectrum selection decision-making is executed. In this case, the belief vector always is computed with $m^{(i)}=0$ to capture the exact interference state at time $t$.

(b) Periodic Measurements (PM) strategy: It consists of performing periodic measurements of the $i$ th SB with observation period $T_{o b s}^{(i)}$. In this manner, the elapsed time $m^{(i)}$ between the last observation of the $i$ th SB and the decision-making time $t$ will always be upper-bounded by $m^{(i)}<T_{o b s}^{(i)}$.

(c) Steady-state (StS) strategy: It is the simple case in which no actual observations are performed. In this case, it can be easily proved making use of the properties of the ergodic discrete time Markov processes that the values of the belief vector will be equal to the steady-state probabilities [46].

The authors in [45] have introduced the belief-based decisionmaking concept for spectrum selection that can be particularized for the considered observation strategies relying only on simulation analysis. In particular, the achieved results have assessed the impact of the environment dynamics in terms of the traffic generation patterns in the observation strategies supporting the decision-making solution. Results have demonstrated that, for long durations of the data transmission sessions, the StS strategy that does not require dynamic observations becomes the best approach. In turn, for short session durations, the use of the PM solution achieves a good tradeoff between reward and number of measurements for large session generation rates, while for low session generation rates, the use of the $I M$ approach at the decision-making time becomes the most adequate solution. The spectrum selection strategy has also been assessed experimentally in [22] through the real-time testbed illustrated in this paper, evaluating an actual scenario where the best trade-off between performance and measurements requirements has been achieved through an appropriate combination between periodical measurements of the radio environment and statistical characterization of the interference variations (i.e., through the PM observation strategy). Finally, in [23], the authors have firstly compared the belief-based decision-making approach against other state-of-the-art solutions to demonstrate the effectiveness of this spectrum selection strategy. Then, the real-time platform presented in this paper enabled carrying out an experimental evaluation to assess practical aspects related to the dynamic variations of the considered interference sources, demonstrating the robustness of the proposed approach when facing non-Markovian interference dynamics.

The different studies illustrated above have been evaluated through specific functionalities provided by the testbed, which have been particularly exploited for the specific study carried out. Therefore, the first aim of this work is to present the main design and 
implementation approaches that allowed the development of the frameworks able to make possible both all the previous described research problems and new innovative spectrum management strategies with a flexible, complete and user-friendly evaluation platform. Then, motivated by the satisfactory published results, the second aim of this paper is to assess definitively the capability of the belief-based spectrum selection solution implemented in the testbed for a real entertainment application in a realistic DH scenario through evaluation of the user perceived end-to-end QoE.

\section{Testbed architecture}

This section provides a complete overview of the testbed architecture, including in-depth details regarding the implementation of each node, the developed functionalities and their applicability.

\subsection{General overview}

The testbed is composed of several reconfigurable nodes, and it has been designed to provide a real-time platform that emulates different CRNs through a centralized cognitive management FA inspired from [16-18]. It has been envisaged and implemented particularly for developing and assessing innovative spectrum management solutions. The testbed design consists of both the development of the nodes of the CRN and the topology of the testbed layout. Considering the centralized nature of the implemented FA, the heart of the testbed is the infrastructure node, which is made of different entities enabling the development of the strategies for spectrum management and that will be analysed in-depth throughout this section. The other testbed nodes represent user terminals that require a spectrum resource for a certain data transmission and several interference sources, which can be configured to transmit at different frequencies. All the networks emulated through the testbed and presented in Section 3 rely on the infrastructure node providing the solutions for spectrum management; moreover, further nodes emulating terminal users and interference sources are needed to assess the spectrum management strategies provided by the infrastructure. For instance, in $[20,21]$, two terminal nodes to emulate a radio link for data transmission and a further node representing an interference source are considered, while in [22,23], two further nodes emulating interference sources are taken into account. Hence, an advantage of this implementation is its scalability, which allows extending the platform through the addition of new interference sources or terminals nodes.

All the testbed nodes can be controlled by a user-friendly interface that allows setting certain input parameters, as will be explained in the following. Signalling flows among nodes can be exchanged through either wireless channels or Ethernet cables. The testbed execution generates a number of statistics that are stored in files so that they can be post-processed later on. In particular, statistics related to the performance obtained in the communication through the radio link can be processed, such as the throughput and the achieved reward in the $j$ th radio link, using the $i$ th SB when it is in state $S^{(i)}=k$ and defined as $r_{j, k}^{(i)}$. The formulation of the reward can reflect different metrics; for instance, in $[22,23,45]$, it is based on the fittingness factor introduced in [19], which is a metric between 0 and 1 and captures how suitable a specific SB is for a specific radio link in terms of bit rate. In detail, based on the fittingness factor formulation defined in [19], the considered reward is given by:

$r_{j, k}^{(i)}=\frac{1-e^{-\frac{\Gamma \cdot U_{j, i, k}}{(\xi-1)^{1 / \xi}\left(R_{j, i, k} / R_{\text {req }, j}\right)}}}{\lambda}$

where $R_{\text {req } j}$ is the bit rate requested for the activation of the $j$ th radio link; $R_{j, i, k}$ denotes the achievable bit rate by the $j$ th radio link in the $i$ th SB given that it is in state $S^{(i)}=k ; \Gamma$ and $\xi$ are shaping parameters with the aim of capturing different degrees of elasticity with respect to the bit rate requirements; $U_{j, i, k}$ is the following utility function that relates the achievable and the required bit rates:

$U_{j, i, k}=\frac{(\xi-1) \cdot\left(R_{j, i, k} / R_{\text {req }, j}\right)^{\xi}}{1+(\xi-1) \cdot\left(R_{j, i, k} / R_{\text {req }, j}\right)^{\xi}}$

and $\lambda$ is a normalization factor given by:

$\lambda=1-e^{-\frac{\Gamma}{(\xi-1)^{1 / \xi}+(\xi-1)^{(1-\xi) / \xi}}}$

Notice that both the fittingness factor and the reward mentioned throughout this paper and considered in the referenced publications [20-23] and [45] are computed through Eq. (1).

\subsection{Hardware and software}

Each node is implemented through Ettus USRP version 1 integrated boards [47] controlled by a personal computer (PC) running the Linux operating system (OS) where GNU radio [48] software, for properly configuring the transmission and reception parameters of the USRP modules, is implemented.

USRP incorporates Analog to Digital and Digital to Analog Converters (ADC/DAC), a Radio Frequency (RF) front end, an FPGA and a USB 2.0 interface to connect to the PC. A typical setup of the USRP board is illustrated in Fig. 2, and it consists of one motherboard that supports up to four daughterboards, where up to 2 receivers and up to 2 transmitters can be plugged in. RF front ends are implemented on the daughterboards.

In detail, the motherboard contains 4 high-speed 12-bit ADCs and 4 high-speed 14-bit DACs. All the ADCs and DACs are connected to the FPGA, which performs high-bandwidth math procedures such as filtering, interpolation and decimation. The DACs' clock frequency is $128 \mathrm{Msample/s,} \mathrm{while} \mathrm{the} \mathrm{ADCs} \mathrm{work} \mathrm{at} 64 \mathrm{Msample/s}$ to digitize the received signal. There exist different types of daughterboards that allow very high USRP reconfigurability. A complete list of daughterboards that can be used with the USRP motherboard can be found in [47], while in the testbed proposed in this paper, XCVR2450 transceivers working in the frequency ranges $2.4-2.5 \mathrm{GHz}$ and $4.9-$ $5.9 \mathrm{GHz}$ have been used.

GNU radio software is a free and open-source toolkit that provides a library of signal processing blocks, such as modulators, demodulators, filters, etc., for building SDRs. It is an empowering tool that enables exploring new ways of using the electromagnetic spectrum and has grown into a widely used cross-platform package that supports SDRs. In GNU radio, the programmer builds a SDR by creating a graph where the vertices are signal processing blocks and the edges represent the data flow between them. All the signal-processing blocks are written in $\mathrm{C}++$; these blocks process streams of data from their input port to their output one. The input and output ports of a signal process block are variable; hence, a block can have multiple outputs and multiple inputs. The Python programming language is used to create a network or graphs and glue the signal processing blocks together [48].

Fig. 3 illustrates a general scheme of the testbed nodes made in this case by a USRP connected to a laptop through a USB cable and that may act as transmitter and receiver, respectively, reflecting the transmission and reception processes and the connection of the PCs running GNU radio software to the hardware platforms. Each node can provide: (1) spectrum sensing functionality exploited by several processes implemented in the infrastructure node and explained in the next subsections; and (2) data transmission, which enables sending either signalling messages among nodes or user data between a pair of terminals. Data transmission functionality can be exploited also by the nodes in charge of provoking the interference; in fact, they can be configured to transmit in certain central frequencies loaded according to the input configuration. 


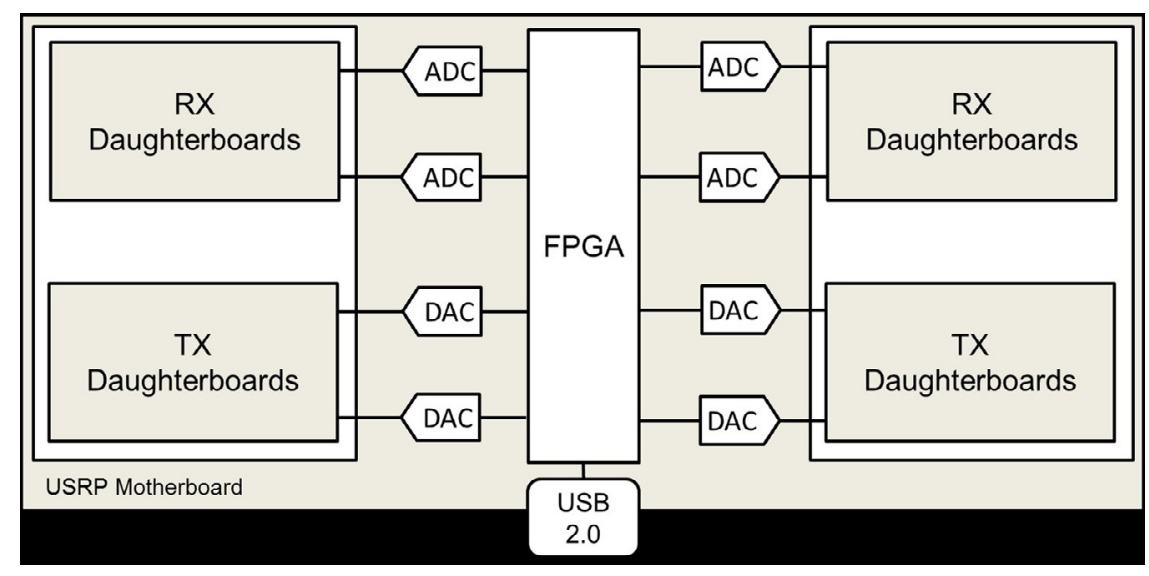

Fig. 2. USRP board.
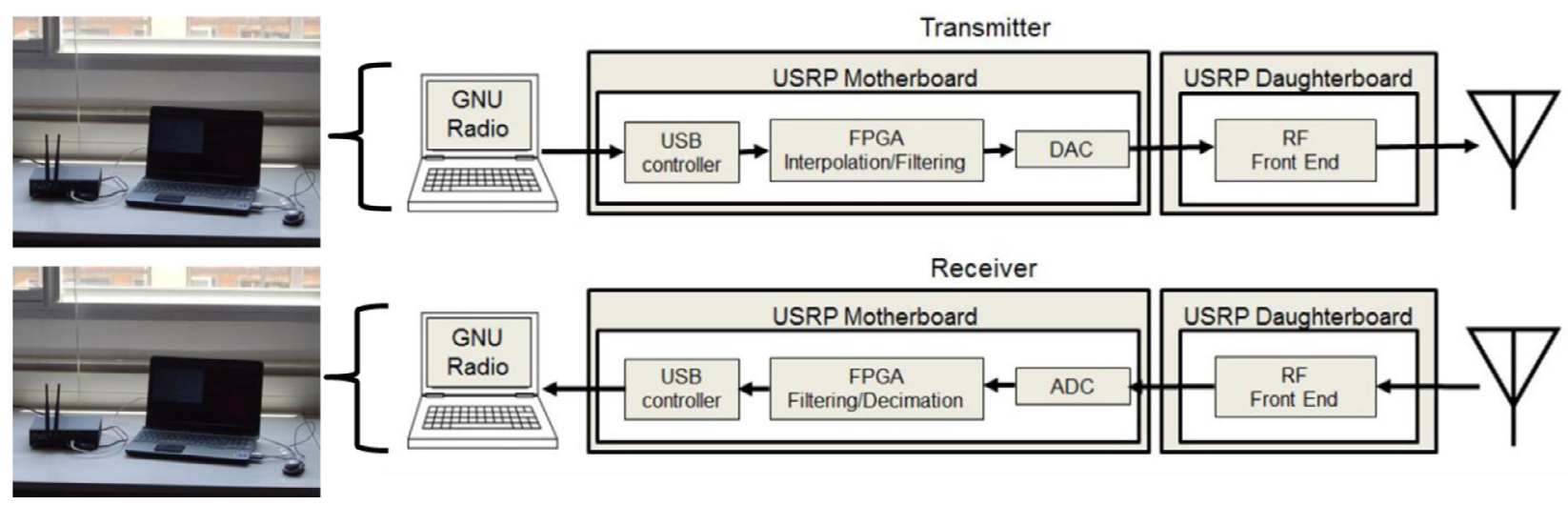

Fig. 3. Transmitter/receiver nodes implemented through USRP and GNU radio.

The script usrp_spectrum_sense.py has been considered for the design of the spectrum sensing functionality implemented in the infrastructure node of the testbed; it can be found in the toolkit provided by the GNU radio software. This script has been used as a basic code for implementing a wideband spectrum analyser to properly sense the desired spectrum bands. However, it has been extended to properly sense the spectrum bands considered in the different spectrum management strategies implemented in the infrastructure. The script receives different input parameters from the user, such as: the lowest frequency of the band to be sensed; the highest frequency of the band to be sensed; how long the spectrum sensing functionality is executed in the entire frequency range; the decimation factor that adapts the incoming data rate to the PC computing capabilities; the Fast Fourier Transform (FFT) size parameter that is the number of samples and their bandwidth considered to perform the magnitude analysis of the sensed signal. Then, the script computes the signal energy detected in each sample during the execution of the spectrum sensing functionality. Finally, the output of the script provides the state of the interference detected in each sample. The decision threshold to detect whether a sample is affected by the interference or not could be chosen for an optimum trade-off between the probability of a missed detection $\left(P_{d}\right)$ and the probability of a false alarm $\left(P_{f a}\right)$. Notice that a missed detection occurs when the interference signal is present in the sensed sample and is not detected by the spectrum sensing functionality, while a false alarm occurs when the sensed sample is idle and the spectrum sensing functionality indicates the presence of the interference signal. Notwithstanding, the optimum trade-off between $P_{d}$ and $P_{f a}$ would require knowledge of noise and detected signal interference powers. While the noise power can be estimated, the signal interference one is difficult to estimate because it depends on many varying factors, such as transmission and propagation characteristics. Hence, the threshold is normally set to satisfy a certain $P_{f a}$ [49], which only requires the noise power to be known. Therefore, the threshold considered in the script and implemented in the testbed is set as the measured noise plus a margin defined to reduce $P_{f a}$; in detail, it has been selected using the following procedure in accordance with [50]: (i) to provide an estimate of the thermal noise, the USRP antenna was replaced with a matched load (i.e., a 50-Ohm resistor), and measurements were performed in the bands from 2.4 to $2.5 \mathrm{GHz}$ and from 4.9 to $5.9 \mathrm{GHz}$; (ii) the Cumulative Distribution Function (CDF) of the thermal noise was calculated for the samples; and (iii) a threshold between the thermal noise and signal energy was selected considering $P_{f a}$ equal to $1 \%$.

The data transmission functionality has been implemented through the GNU radio's benchmark_tx.py and benchmark_rx.py scripts. In detail, the file benchmark_tx.py is the transmitter code that generates packets whose size is specified by the user, while the file benchmark_rx.py is the receiver code, which listens for incoming packets and checks for errors in each received packet through the Cyclic Redundancy Check (CRC) error-detection code. These scripts take the following input parameters from the users: a modulation scheme between the Gaussian Minimum Shift Keying (GMSK) and the Differential Binary Phase Shift Keying (DBPSK); the data transmission bit rate; the central frequency of the SB for the data transmission; and the packet size only for the script benchmark_tx.py.

The main problem found in these scripts is that the implementation uses only a one-way data flow; therefore, the transmitter cannot receive ACK (positive acknowledge) or NACK (negative acknowledge) messages, which are useful for allowing retransmissions of either lost or erroneous packets. Hence, these scripts have been modified by 


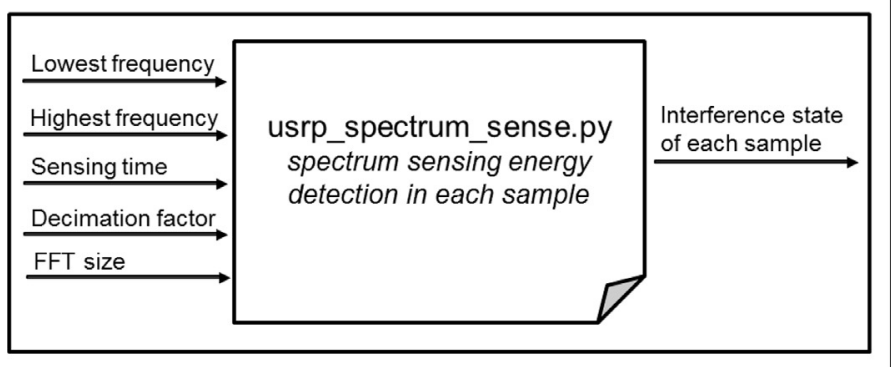

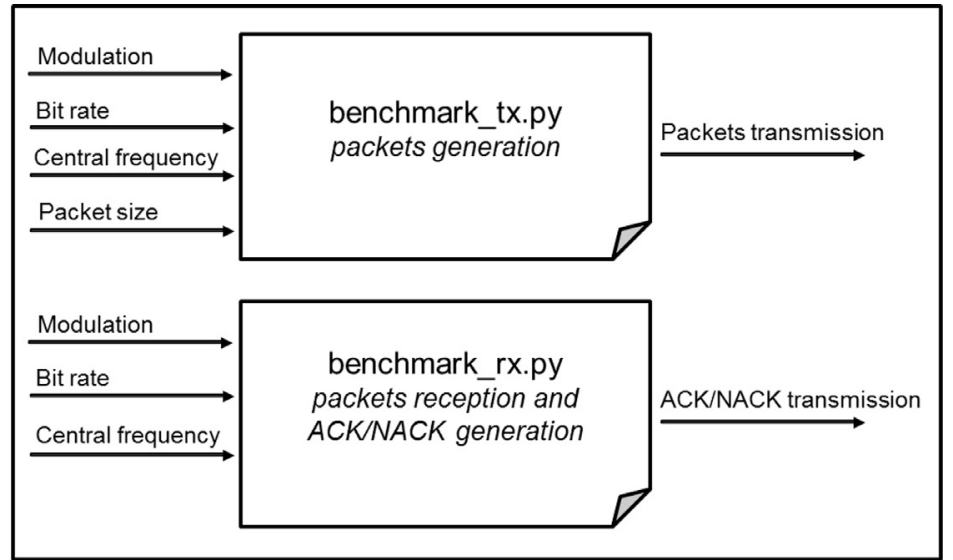

Fig. 4. Input/output scripts parameters. adding a stop and wait error-control method that uses acknowledgement messages to monitor the performance of the data transmission. Fig. 4 summarizes the general working principles of the scripts implemented in the testbed node.

\subsection{Infrastructure node}

As previously mentioned, the heart of this testbed is the infrastructure node implemented through a USRP controlled by a PC running the scripts previously introduced. The FA of the infrastructure node is depicted in Fig. 5. It controls all the decision-making processes implemented in the platform and consists of the main entities illustrated in the next subsections.

\subsubsection{Dynamic spectrum management}

It provides strategies that enable finding radio spectrum opportunities that guide the spectrum selection decision-making in the context of ONs. Hence, this functionality allows the infrastructure to obtain the necessary awareness level in its environment to determine spectrum availability and to make the appropriate decisions during the decision-making spectrum selection for ONs. Awareness of the radio environment is achieved through the exploitation of the spectrum sensing functionality implemented in the USRP. Hence, this block implements the spectrum opportunity identification solution for ONs that give the signal energy of each SB, establishing whether it is free of interference. This entity is deactivated for the spectrum management solutions based on the belief vector.

\subsubsection{Knowledge management}

It is composed of the Knowledge Manager (KM) and the Knowledge Database (KD). The KM is responsible for computing and storing statistical information of the radio environment in the KD achieved through the Context Awareness (CA) functionality illustrated later on and for assisting in the spectrum selection decision-making process. In particular, for the solutions envisaged for ONs, the KM supports the CMON during the decision-making process, storing in the KD the channel occupancy information from past time periods. Concerning the strategies based on the belief vector, it stores in the KD all the information regarding state transition probabilities, steady-state probabilities, reward values based on the fittingness factor, belief vectors and average session durations of data transmission. Notice that the $\mathrm{KM}$ is also in charge of computing the belief vector based on the information stored in the KD. In detail, the belief vector $\mathbf{b}^{(\mathbf{i})}$ at time $t-m^{(i)}$ (i.e., when the last observation of the actual interference state was taken in the $i$ th $\mathrm{SB}$ ) is given by:

$\mathbf{b}^{(\mathbf{i})}\left(t-m^{(i)}\right)=\mathbf{x}\left(S^{(i)}\left(t-m^{(i)}\right)\right)$ where $\mathbf{x}(k)$ is defined as a column vector of $K+1$ components numbered from 0 to $K$ that has all of them equal to 0 except the $k$ th component, which is equal to 1 . Then, the belief vector at a time instant $t>t-m^{(i)}$ can be obtained at the previous time step $t-1$ using the state transition probability matrix $\mathbf{P}^{(\mathbf{i})}$ stored in the KD as follows:

$\mathbf{b}^{(\mathbf{i})}(t)=\mathbf{b}^{(\mathbf{i})}(t-1)\left[\mathbf{P}^{(\mathbf{i})}\right]$

By recursively applying Eq. (5) for the last $m^{(i)}$ time steps and making use of Eq. (4), the belief vector at time $t$ as a function of the last observation is given by:

$\mathbf{b}^{(\mathbf{i})}(t)=\mathbf{b}^{(\mathbf{i})}\left(t-m^{(i)}\right)\left[\mathbf{P}^{(\mathbf{i})}\right]^{m^{(i)}}=\mathbf{x}\left(S^{(i)}\left(t-m^{(i)}\right)\right)\left[\mathbf{P}^{(\mathbf{i})}\right]^{m^{(i)}}$

\subsubsection{Decision-making}

This functionality executes the selection of the most appropriate radio SB whenever requested by a radio link in accordance with the statistic information stored in the knowledge management. It also triggers the execution of the measurements of the radio environment made by the CA. In particular, the solution presented for ONs and implemented in the CMON is based on the following procedures: (i) it estimates the fittingness factor for each link and available SB based on the information stored in the KD that is maintained with different past SB occupancy information; (ii) it selects the SB guaranteeing the highest fittingness factor for the radio link. The proposed spectrum selection strategy based on the belief vector implements the decision-making that maximizes the expected reward and that can be particularized for the observation strategies introduced in Section 3. In detail, in this block, the following functionalities are implemented: (i) the observation strategy decision-making in charge of selecting one of the observation strategies among the IM, the PM and the StS to be applied in the $i$ th SB depending on the traffic generation patterns and the interference behaviour; and (ii) the spectrum selection decisionmaking in charge of selecting an SB each time that a new session is established in the $j$ th radio link particularized for the selected observation strategy. Moreover, it targets the maximization of the expected reward that the session will experience along its duration $D_{j}$ at future time instants $t+n$ relying on the belief vector, which can be achieved recursively through $\mathrm{Eq}$. (5), leading to:

$\mathbf{b}^{(\mathbf{i})}(t+n)=\mathbf{b}^{(\mathbf{i})}(t)\left[\mathbf{P}^{(\mathbf{i})}\right]^{n}$

Then, the spectrum selection decision-making policy is formulated as:

$S B_{j}(t)=\arg \max _{i} \frac{1}{\bar{D}_{j}} \sum_{n=1}^{\bar{D}_{j}} \mathbf{b}^{(\mathbf{i})}(t+n) \mathbf{r}_{j}^{(\mathbf{i})}$

where $\mathbf{r}_{j}^{(\mathbf{i})}$ represents the reward vector of the $j$ th link in the different interference states of the $i$ th SB and $\mathbf{b}^{(\mathbf{i})}(t+n)$ is achieved by Eq. (7). 

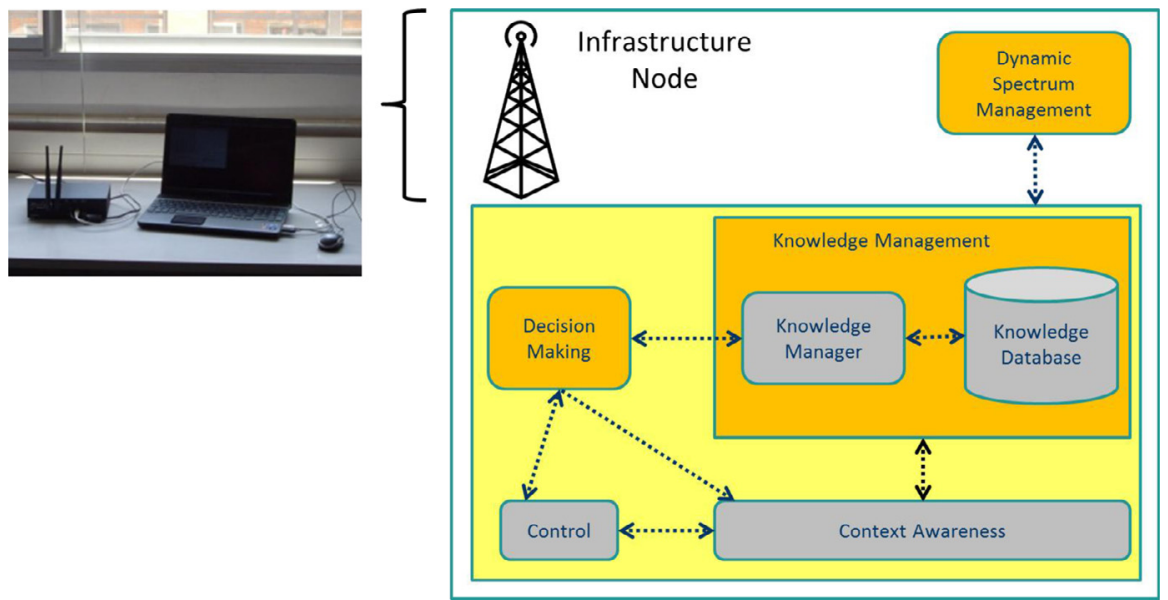

Fig. 5. FA of the infrastructure node.

Notice that $\mathbf{b}^{(\mathbf{i})}(t)$ in Eq. (7), which is computed through Eq. (6) by the $\mathrm{KM}$, provides at the decision-making time $t$ the future evolution of the reward in each of the SBs until the session end, exploiting past measurements of the interference state together with the statistical characterization of the interference dynamics. Finally, given that the session duration $D_{j}$ is typically unknown at the decision-making time $t$, it is assumed to be characterized statistically in terms of its average value $\bar{D}_{j}$.

\subsubsection{Context awareness}

It executes measurements of different parameters triggered by the decision-making when needed, which characterize the radio environment, and carried out through the spectrum sensing functionality implemented in the USRP. In detail, in the case of solutions for ONs, it provides both feedback of the QoS experienced by the nodes of the ON during the ON maintenance phase and channel occupancy information. In the case of strategies based on the belief vector, it provides the observations through measurements of the radio environment that allow the KM to update the belief vector when needed. Furthermore, in [22], the spectrum sensing functionality of the USRP is exploited to enable the CA functionality for validating the dynamic variation of the interference sources.

\subsubsection{Control}

It is implemented also in the users' terminal nodes constituting the testbed, and it is in charge of handling the message exchange between the terminals and the decision-making to support the establishment/release of radio links. Moreover, this entity can exchange the necessary signalling messages with any node that requests the CA measurements of the radio environment. In the case of an $\mathrm{ON}$, it triggers the $\mathrm{ON}$ creation to the decision-making and, after that, whether to proceed with an ON reconfiguration decided during the maintenance phase. In this case, signalling messages flows are implemented through the Control Channel for the Cooperation of the Cognitive Management System (C4MS) protocol, which uses the implementation option based on IEEE 802.21 "Media-Independent Handover (MIH) Services" [51]. As an example, the interactions among the relevant functional blocks of the testbed following the C4MS protocol for the ON creation are illustrated in Fig. 6. This example illustrates the signalling messages exchanged among the control entity of two terminals forming the $\mathrm{ON}$ (i.e., $T 1$ and $T 2$ in the figure), which need an SB to transmit data in the ON link, and of the infrastructure (i.e., $I$ in the figure) that executes the spectrum opportunity identification and the spectrum selection functionalities. The first step towards the ON Creation (ONC) is the ON Negotiation (ONN), which allows obtaining the information used for the configuration of the radio link. Hence, the message ONN.request defined to start the negotiation is sent from $T 1$ to $I$ to obtain a valid configuration of the radio link. The message indicates the terminals involved (i.e., $T 1$ and $T 2$ ) and the QoS requirements that the link is expected to support in terms of the required bit rate. Then, I sends an ONN.request to T2, informing it about the intention to establish a direct radio link with $T 1$ and allowing it to join the negotiation process for the derivation of the radio link configuration. $T 2$ replies to I with an ONN.response message, notifying it of its acceptance for the establishment of the link. The control entity in $I$ inquires the DSM to determine spectrum availability for the link, sending an SB_availability.request message. Therefore, the spectrum opportunity identification strategy is executed (i.e., during SOI execution in the figure). The DSM provides the decision-making with the available SBs. Then, the decision-making sends a message to the knowledge management to achieve past SB occupancy information (i.e., SB_info.request in the figure). Once this information is obtained, the spectrum selection strategy is executed to decide the SB guaranteeing the highest fittingness factor to be allocated to the link (i.e., during SS execution in the figure). The proposed selected SB is sent from the decision-making to the control block in the Selected_SB.info message, and then it is transferred to $T 1$ by issuing an ONN.response message. Therefore, the ONC can be started, and $T 1$ sends the message ONC.request defined to start the creation to I with the final ON configuration with the selected SB. I sends another ONC.request towards $T 2$ with the final ON configuration. $T 2$ replies with an ONC.response message with a successful result-code, indicating that the terminal is ready to establish the link. I concludes the ON creation procedure by sending an ONC.response message to T1. The link establishment between $T 1$ and $T 2$ takes place at this point. Notice that the CA entity is not involved during the ONC phase. In fact, it is in charge of providing either SB occupancy information to the knowledge management before the decision to create an ON or the feedback of the QoS experienced by the nodes of the $\mathrm{ON}$ during the $\mathrm{ON}$ maintenance phase.

In the case of strategies based on the belief vector, the control entities of each node allow signalling messages to be exchanged through Ethernet cables. In this case, signalling message flow protocols are defined by the authors. Fig. 7 illustrates an example of interactions among the relevant functional blocks of the testbed during the decision-making spectrum selection based on the belief vector.

The spectrum selection functionality illustrated in the figure is executed to allow a link establishment between terminals $T 1$ and $T 2$ requiring an SB and supposing a previous selection of the $I M$ observation strategy. A detailed explanation of the selection of the most appropriate observation strategy depending on the environment dynamics in terms of traffic generation patterns and interference behaviour can be found in $[23,45]$. Notice that, considering the IM 


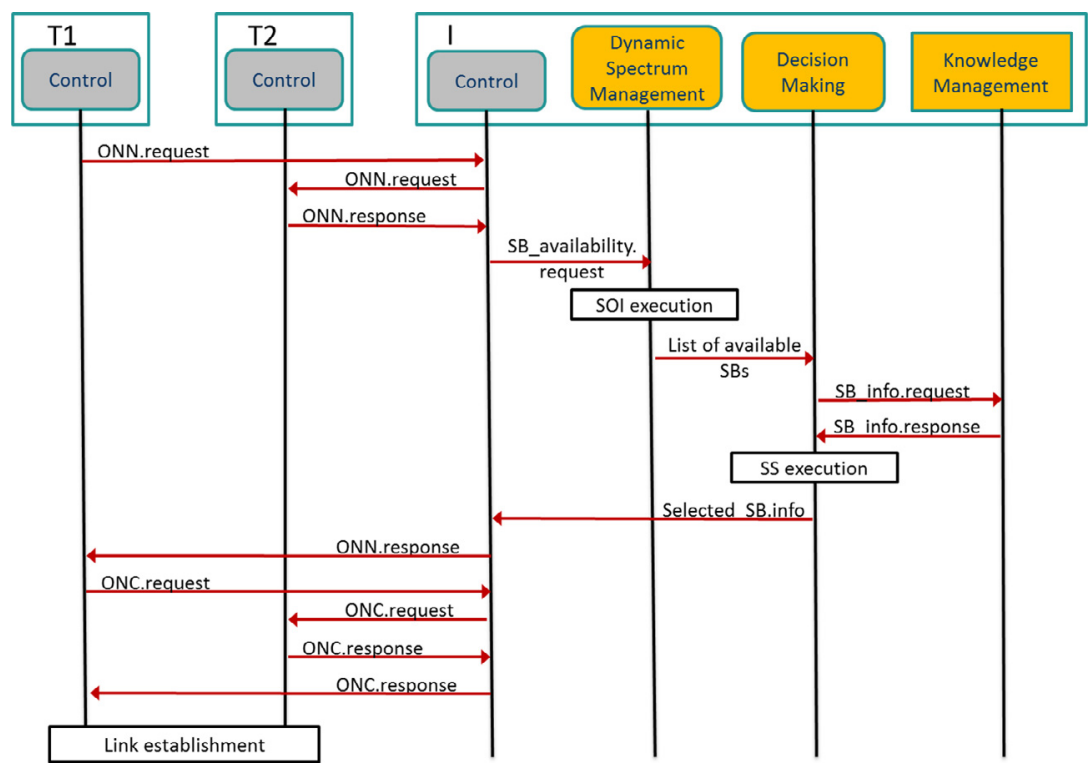

Fig. 6. Implemented message exchange for the ON creation.

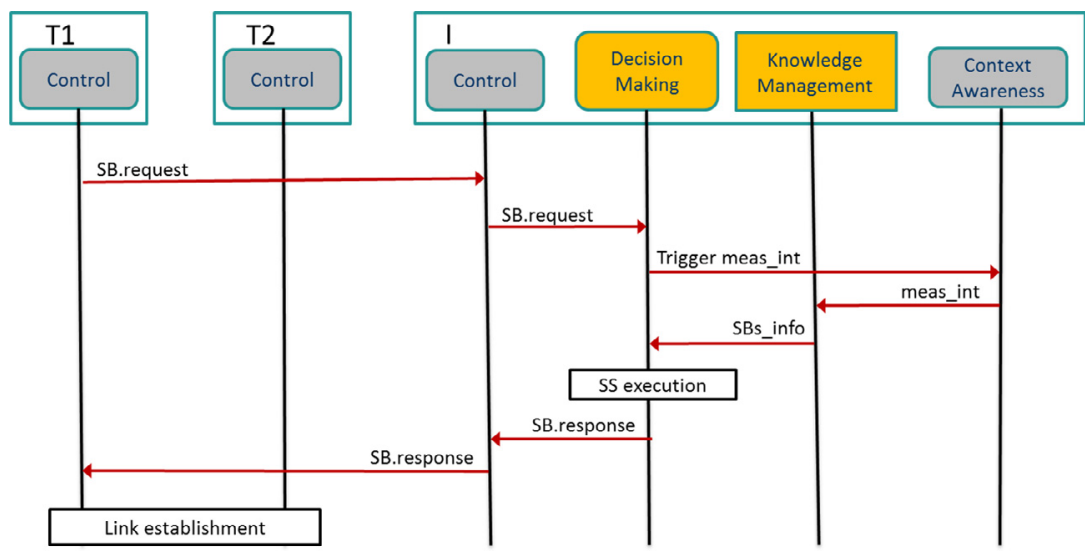

Fig. 7. Implemented message exchange for belief vector-based spectrum selection.

observation strategy, the belief vector in Eq. (6) is computed with $m^{(i)}=0$, and therefore it captures the exact interference state at the decision-making time $t$. Then, the belief vector at $t$ is given by:

$\mathbf{b}^{(\mathbf{i})}(t)=\mathbf{x}\left(S^{(i)}(t)\right)$

Focusing now on the messages exchanged among the nodes, the request for an $\mathrm{SB}$ is sent from one of the terminals (i.e., $T 1$ in this example) to the control block of the infrastructure, and then it is delivered to the decision-making by the SB.request message. As the observation strategy previously selected is the $I M$, the decision-making sends the message that triggers the measurement of the interference states in all the SBs (i.e., the Trigger_meas_int message in the figure) to the CA. Then, the CA provides by the meas_int message the interference states through measurements of the radio environment that allow the KM in the knowledge management to update the belief vector through Eq. (9). The values of the updated belief vectors are then sent in a message from the knowledge management to the decision-making together with the transition probabilities matrices, the rewards of each SB and the statistical characterization of the data transmission duration stored in the KD (i.e., the SBs_info message in the figure). Once the SB information is obtained, the spectrum selection strategy is executed (i.e., during SS execution in the figure) following Eq. (7) and Eq. (8). Finally, the selected SB is sent from the decision-making to the control block, and then it is delivered to $T 1$ in the SB.response message. At this point, the link establishment can be carried out between the terminals.

\section{A digital home application}

The aim of this section is to illustrate the practicability of the proposed testbed in terms of user-perceived end-to-end QoE through an application in the realistic environment of a real DH scenario. A future DH system is expected to consist of different types of devices, such as equipment with communication capabilities (e.g., desktop PCs and laptops), consumer electronics (e.g., TV sets with wireless interfaces, digital media servers, game consoles, home security and automation systems), and more traditional appliances equipped with communication interfaces to allow, for example, remote control and monitoring (e.g., washing machines and fridges). Moreover, the provisioning of wireless management applications in the DH requires an efficient exploitation of all possible sources of available spectrum resources, such as license-exempt ISM bands and UHF bands (i.e., TV white spaces) through, e.g., the European association for standardizing information and communication systems (ECMA)-392 radio networking standard [52], and also the exploitation of the licensed spectrum (e.g., the spectrum licensed to a mobile network operator providing management services in the $\mathrm{DH}$ ) as a mechanism for enhancing QoS provision to some DH connections. 


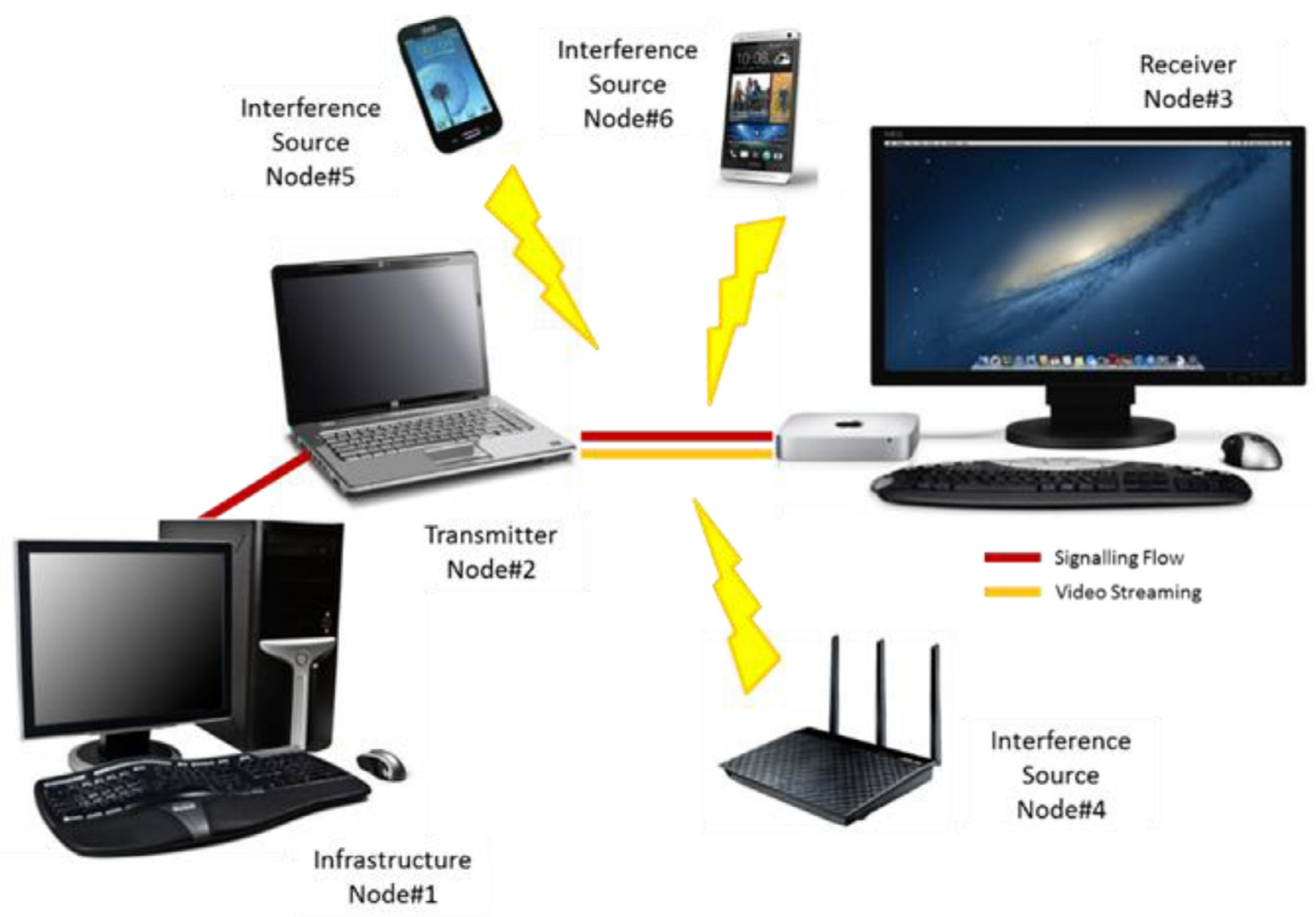

Fig. 8. Emulated digital home scenario.

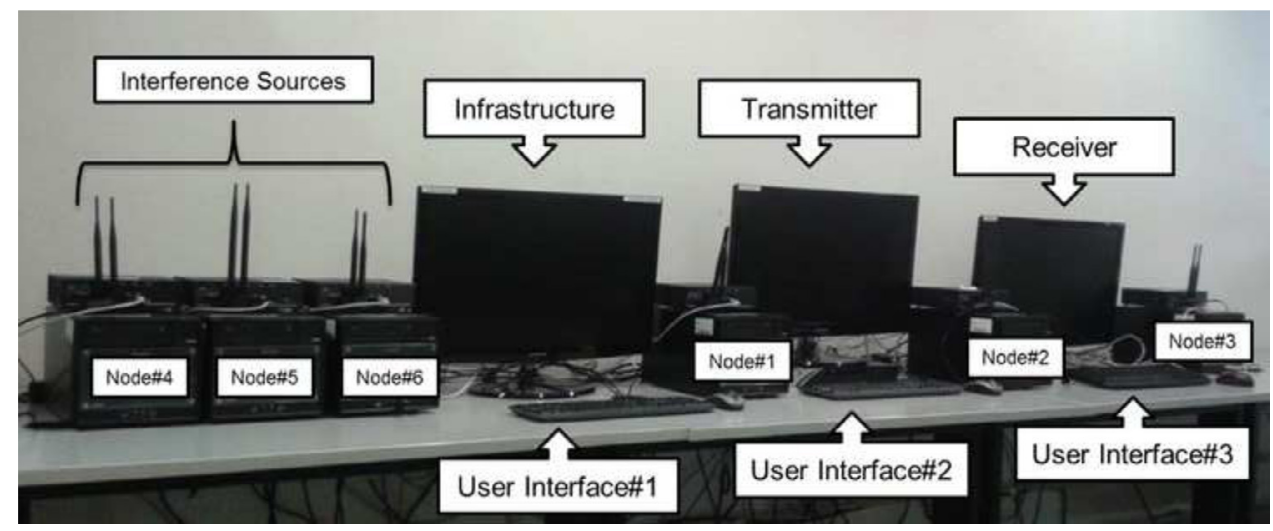

Fig. 9. Testbed configuration.

In the context of this section, the emulation of the DH scenario illustrated in Fig. 8, where an entertainment application is provided, has been implemented through the testbed presented in this paper. In detail, Node\#2 and Node\#3 in the figure represent two PCs that need an SB selected by the infrastructure (i.e., Node\# 1 in the figure) for the transmission of a streaming video. After the selection of the SB, the video is transmitted by Node\#2, where it is stored, to Node\#3, which is connected to a large screen where the video is displayed. Moreover, Nodes \#4, \#5 and \#6 are possible external interference sources transmitting in the SBs considered for this evaluation. To emulate this scenario through the testbed, six reconfigurable nodes made of a USRP connected to a PC have been considered and illustrated in Fig. 9.

In Node\#1, which emulates the infrastructure depicted in Fig. 5, the decision-making, the knowledge management, the CA and the control functionalities are implemented. In the decision-making, the spectrum selection policy based on the belief vector presented in $[22,23,45]$ is considered; further details of this implementation are provided in the next subsection.
Moreover, Node\#2 and Node\#3 exploit the data transmission functionality provided by the USRP for the streaming video. As seen in Fig. 9, three screens are the user interfaces that allow running and controlling the testbed operation. Specifically, User Interface\#1 is connected to a switch that allows configuring the parameters of the interference sources and the infrastructure, while User Interface\#2 allows configuration of the transmitter that will send the streaming video. Finally, User Interface\#3 enables configuration of the receiver connected to the screen where the video will be displayed.

\subsection{Configuration}

A set of 3 SBs centred, respectively, at the frequencies 5472, 5490, and $5508 \mathrm{MHz}$ are taken into account. Two different interference states are considered for each $i$ th SB: $S^{(i)}=0$ when no interference exists and $S^{(i)}=1$ when the interference corresponds to its maximum value. The durations of the interferences states for each SB emulated, respectively, by Nodes \#4, \#5 and \#6 are exponentially distributed 
Table 1

Characterization of the interference states.

\begin{tabular}{lll}
\hline Spectrum block & State $S^{(i)}=0$ (time steps) & State $S^{(i)}=1$ (time steps) \\
\hline$\# 1$ & $600(100 \mathrm{~min})$ & $200(33 \mathrm{~min})$ \\
$\# 2$ & $170(28 \mathrm{~min})$ & $650(108 \mathrm{~min})$ \\
$\# 3$ & $600(100 \mathrm{~min})$ & $400(66 \mathrm{~min})$ \\
\hline
\end{tabular}

with the average times presented in Table 1 , considering that the interference sources operate in time steps of $10 \mathrm{~s}$ (e.g., 600 time steps in the table correspond to $100 \mathrm{~min}$ ). A radio link is considered to transfer the streaming video, whose duration $D$ is approximately $20 \mathrm{~min}$, from Node\#2 to Node\#3 with a bit rate requirement of $R_{\text {req }}=1 \mathrm{Mbps}$. Node\#2 implements a packet segmentation that allows for division of the video into Ethernet frames whose size can be selected during the configuration set-up of the testbed.

Considering the durations of the interference states represented in Table 1 and the duration of the video, the most appropriate observation strategy at the decision-making time selected by the observation strategy decision-making is the IM that allows achieving the best trade-off between performance results and measurement requirements in this scenario. In fact, this is a case characterized by short session duration compared to the interference duration states and low session generation rates, which implies the use of the IM observation strategy; for more details about the metrics that define short session duration and low session generation rate, the reader is referred to [45].

Hence, at the decision-making time, the infrastructure selects for the video transmission an SB executing the decision-making criterion that maximizes the expected reward during the overall streaming video transmission, relying on the belief vector and particularized to the IM observation strategy. The KD stores the information regarding state transition probabilities considering the configuration of the SBs illustrated in Table 1 to update the belief vectors, reward values achieved in each SB considering its interference states, and streaming video session duration. The reward considered in this paper ranges between 0 and 1 , where 0 means that the $i$ th SB is in interference state $S^{(i)}(t)=1$ and 1 means that it is free of interference, i.e., $S^{(i)}(t)=0$. The KM is in charge of computing the belief vector based on the information stored in the KD and the measurements information received by the CA.

Specifically, the CA entity of the infrastructure allows performing measurements of the interference states of each SB by means of the spectrum sensing functionality implemented in the USRPs and is triggered by the decision-making when the streaming video has to be executed. Moreover, during the streaming video session, Node\#2 is programmed to compute the average reward value in the selected $i$ th SB. Finally, the control entities of each node allow the signalling messages to be exchanged following the protocol for spectrum selection, which relies on the IM observation strategy and is illustrated in Fig. 7.

\subsection{Key performance indicators}

To demonstrate the capabilities of the testbed illustrated in this paper in a realistic DH scenario, the following KPIs are considered:

- Interference behaviour of the SBs: It illustrates the temporal evolution of the interference states that characterizes each SB. This KPI is considered to show how the decision-making spectrum selection approach allows a streaming video transmission in an SB free of interference.

- Received Ethernet frames: It is the percentage of frames received by Node\#3 during the streaming video transmission that consists of the percentage of frames received correctly, the percentage of frames received erroneously and the percentage of frames lost during transmission.
- QoE: It is represented by the resolution of the video displayed on the screen connected to Node\#3.

- Averaged session reward: It is the average reward experienced during a data transmission session depending on the interference state of the allocated SB.

\subsection{Obtained results}

The aim of this section is to assess the capability of the real-time testbed illustrated in this paper for a real entertainment application provided in the DH scenario illustrated in Fig. 8. Considering the configuration previously described, in the infrastructure node, the spectrum selection approach based on the IM observation strategy is executed at the decision-making time; moreover, a random strategy as a baseline reference for performance comparison is also included. To this aim, two different experiments have been carried out.

\subsubsection{Evaluation of the quality of experience}

The first experiment aims to compare the IM-based solution against the random one in terms of Ethernet frames and QoE perceived by the user. Hence, the streaming video transmission has been carried out twice, one per strategy at different times. Fig. 10 illustrates the temporal evolution of the experiment during $15 \mathrm{~h}$, which is implemented as a sequence of consecutive inactivity periods (OFF in the figure) and two activity periods characterized by the video transmission ( $\mathrm{ON}$ in the figure). Each activity period starts with the request of an SB from the transmitter to the infrastructure, which in turn executes either the IM-based spectrum selection strategy or the random one; then, the video is transmitted to the receiver on the selected SB for $20 \mathrm{~min}$. In detail, from the figure, it can be noticed that, after $120 \mathrm{~min}$, the transmitter requests an SB by sending an SB.request message to the control block of the infrastructure node, which executes the decision-making criterion based on the IM observation strategy. After approximately $730 \mathrm{~min}$, the transmitter requests an SB to the infrastructure node that executes the random strategy.

Fig. 11 shows the interference states that characterize each SB (i.e., 0 when no interference exists and 1 when the interference reaches its maximum value) during the $15 \mathrm{~h}$ considered for the experiment; moreover, in the figure, the time instant at which the transmitter requires an $\mathrm{SB}$ for the video transmission and then at which the decision-making selects an SB for each strategy (i.e., the IM and the random) is shown.

This figure illustrates how the infrastructure selects the first SB that guarantees the maximum expected reward during the streaming video transmission in the case of the IM observation strategy. Notice that, at the decision-making time, the second SB is free of interference; notwithstanding, the infrastructure, which follows the decision-making criterion maximizing the expected reward for the overall video transmission, selects the SB that guarantees a streaming video free of the interference during its complete duration of $20 \mathrm{~min}$. Furthermore, from Fig. 11, it can be observed that the infrastructure node selects the second SB for the video transmission following the random strategy; in this case, the selected block is affected by the interference at the decision-making time instant. It is worth mentioning that the decision-making solution implemented in the infrastructure would have selected at that time the third SB following the IM strategy, ensuring a block free of interference for the overall streaming video transmission.

The consequent performance results achieved through each spectrum selection strategy are illustrated in Fig. 12 in terms of received Ethernet frames. From the figure, it can be observed that, in the case of the IM-based strategy, only $0.5 \%$ of the frames have been received erroneously by Node\#3 and $0.03 \%$ of the frames have been lost; although the SB has been free of interference during the overall video transmission, possible spurious uncontrolled interference existing in the realistic scenario can cause the detriment of a few frames. In 


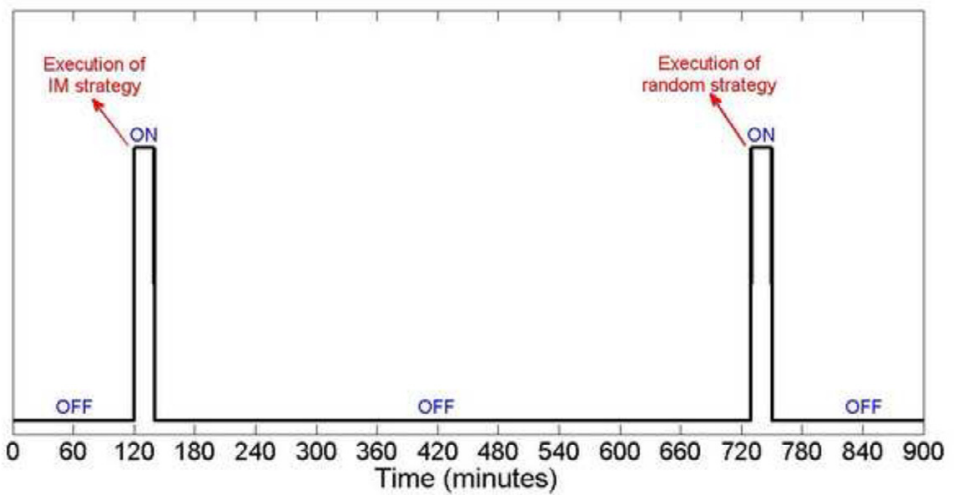

Fig. 10. Temporal evolution of the experiment.
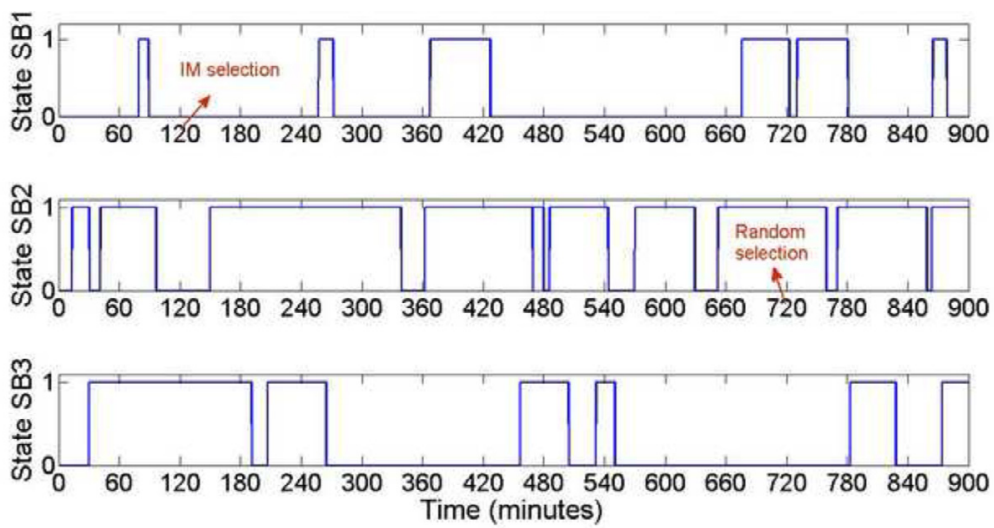

Fig. 11. Temporal evolution of the interference of the spectrum blocks.

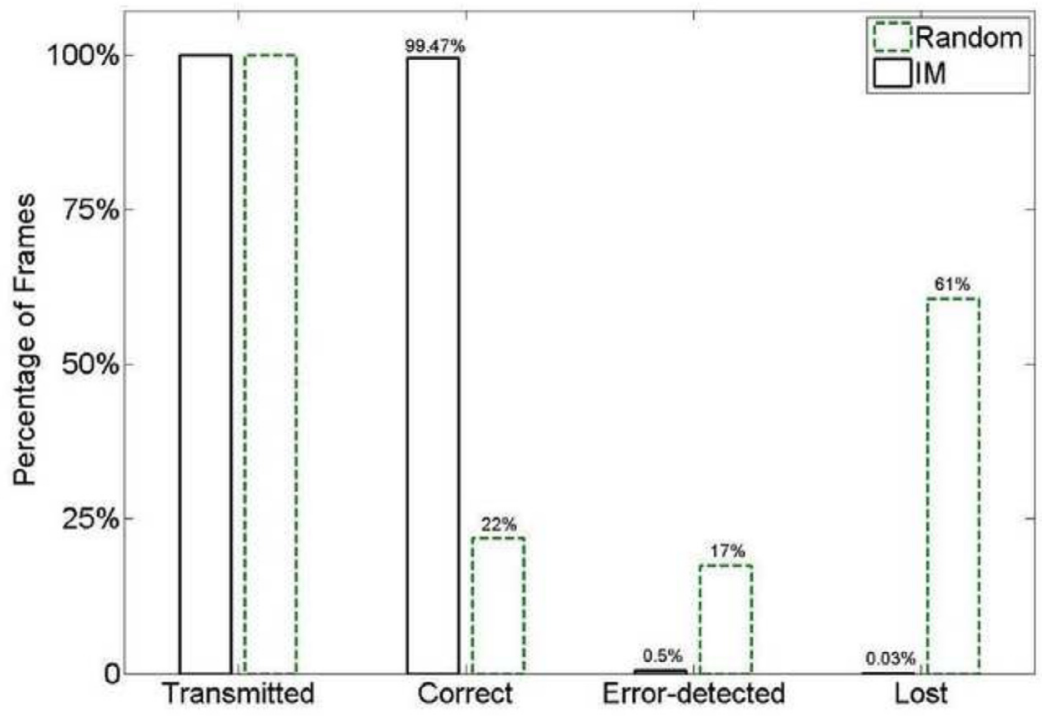

Fig. 12. Transmitted and received frames.

the case of the random strategy, it can be noticed that $17 \%$ of the frames have been received erroneously by Node\#3 and even $61 \%$ of the frames have been lost.

To analyse the impact of the interference on the user-perceived QoE in terms of the resolution of the video displayed on the screen connected to Node\#3, Figs. 13 and 14 illustrate some screenshots of the received frame sequences during two different cases. The first case illustrated in Fig. 13 corresponds to three screenshots of the received video on the SB selected through the IM-based strategy and is free of interference (i.e., SB1). From the figure, it can be observed that the implementation of the proposed observation strategy at the decision-making time allows displaying the video free of the interference. The second case shown in Fig. 14 corresponds to three screenshots of the received video on the SB selected through the random strategy and is affected by the interference (i.e., SB2). In this case, from the figure, it can be noticed a clear degradation of the video resolution. This is due to the massive amount of frames either received erroneously or lost (see Fig. 12). 


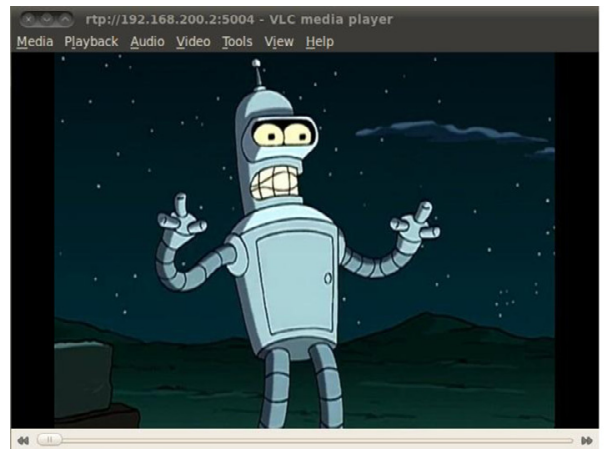

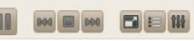

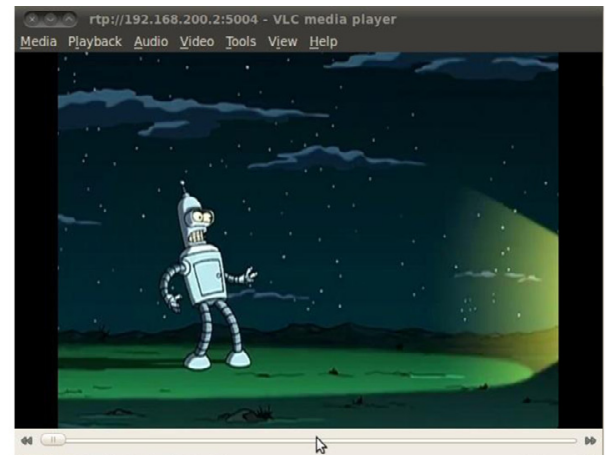

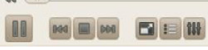

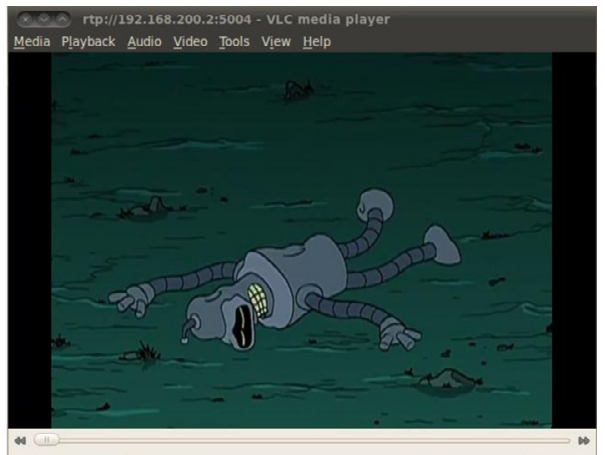

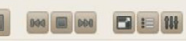

Fig. 13. Screenshots captured during video transmission supported by the IM-based strategy.

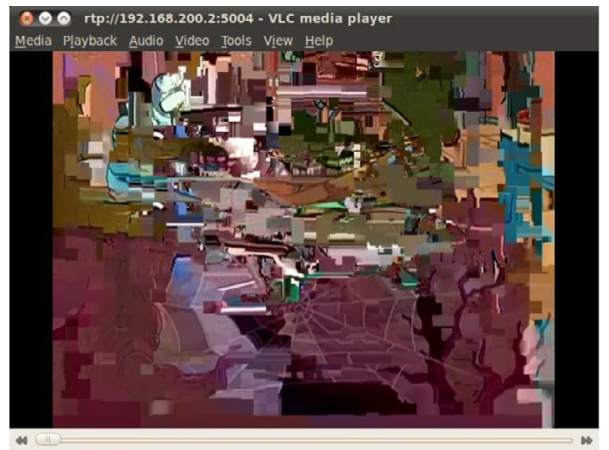

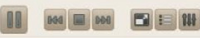

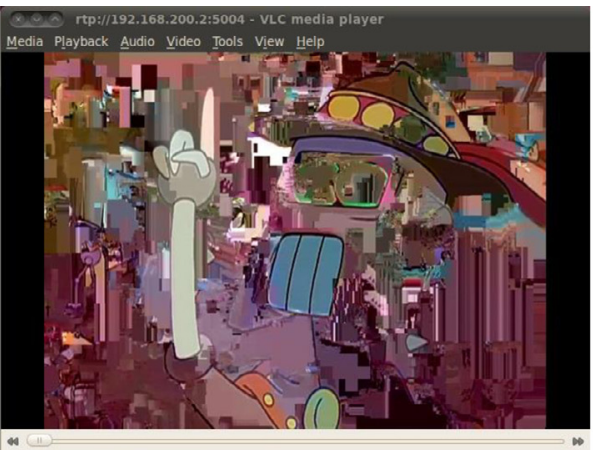

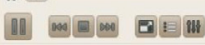

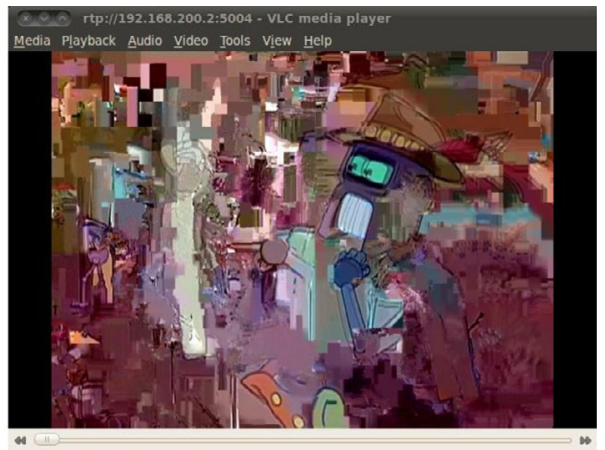

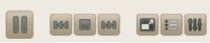

Fig. 14. Screenshots captured during video transmission supported by the random strategy.

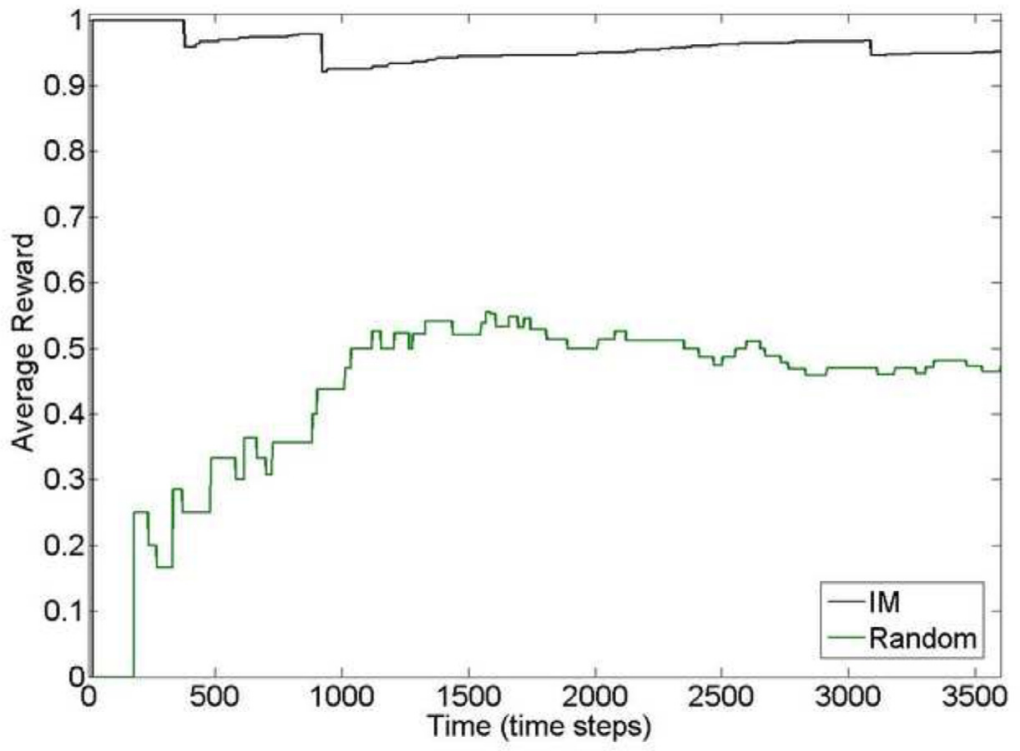

Fig. 15. Performance evaluation in terms of the average session reward.

\subsubsection{Evaluation of the average reward}

The second experiment aims at demonstrating that, with the same hypothesis of the interference sources illustrated in Table 1, the decision-making strategy based on the belief vector can guarantee satisfactory performance results during the $15 \mathrm{~h}$ of emulation time in terms of the average reward compared to the random case. This experiment is implemented as a sequence of consecutive streaming video transmissions of $20 \mathrm{~min}$ and periods of inactivity over $15 \mathrm{~h}$. The time of the inactivity periods is considered to be exponentially distributed with an average of $20 \mathrm{~min}$. Then, the average reward experienced by the link depending on its allocated SB and corresponding interference for each video transmission session has been calculated, averaged over $15 \mathrm{~h}$, and compared for cases of spectrum selection based on both the IM strategy and the random one. The result of this experiment is illustrated in Fig. 15. From the figure, it can be observed that the spectrum selection based on the IM strategy allows a convergence of the averaged session reward to approximately 0.97 , which represents a value $40 \%$ greater than that achieved by the random approach (i.e., in this case, the average session reward converges to 0.58 ). 


\section{Conclusions}

This work has presented a real-time emulation platform for spectrum management in the context of opportunistic networks and more general cognitive radio networks. The aim of this testbed is to provide a highly accurate and realistic platform on which the performance of innovative spectrum management strategies relying on the cognitive radio paradigm can be fully assessed and evaluated before considering them in real systems. Although several analytical models and off-line simulators found in the literature provide powerful tools for obtaining preliminary results, it is also necessary to provide to the research and industrial community real-time platforms that reflect a complete realistic environment. In this context, this paper has accurately described a real-time testbed that enables the emulation of spectrum management strategies with applicability in a wide set of scenarios and use cases exploiting the cognitive radio paradigm.

This paper has provided an in-depth description of the real-time platform, highlighting many interesting implementation details and illustrating its applicability for different solutions developed for opportunistic networks and cognitive radio networks. Moreover, the demonstration of streaming video in a realistic Digital Home (DH) scenario has allowed validation of the effectiveness of the real-time testbed, assessing its practicability in terms of user-perceived end-toend Quality of Experience (QoE) in a realistic environment. As a part of the future work, the extension of the number of the nodes and the potential addition of new entities in the infrastructure node to support novel radio technologies are envisaged. Possible innovative technologies include Device-to-Device (D2D) communications in the context of 5G wireless networks; Unlicensed LTE (U-LTE) technology, which has been proposed as an innovative way to expand LTE spectrum capacity; and energy-efficient CR solutions, which are expected to optimize spectrum sharing and energy efficiencies jointly.

\section{Acknowledgement}

This work is supported by the Spanish Research Council and FEDER funds under a RAMSES (Ref. TEC2013-41698-R) grant.

\section{References}

[1] J. Mitola III, Cognitive radio: an integrated agent architecture for software defined radio (Ph.D. dissertation), KTH Royal Institute of Technology, 2000.

[2] K.G. Shin, H. Kim, A.W. Min, A. Kumar, Cognitive radios for dynamic spectrum access: from concept to reality, IEEE Wirel. Commun. 17 (6)(2010) 64-74.

[3] P. Demestichas, G. Dimitrakopoulos, J. Strassner, D. Bourse, Introducing reconfigurability and cognitive networks concepts in the wireless world, IEEE Veh. Technol. Magazine 1 (2) (2007) 32-39.

[4] R.W. Thomas, D.H. Friend, L.A. Dasilva, A.B. Mackenzie, Cognitive networks: adaptation and learning to achieve end-to-end performance objectives, IEEE Commun Mag. 44 (12) (2007) 51-57.

[5] J. Kephart, D. Chess, The vision of autonomic computing, IEEE Comput. 36 (1) (2003) 41-50

[6] G. Dimitrakopoulos, M. Logothetis, P. Demestichas, Performance evaluation of cognitive management functionality for emergency healthcare applications, in: Proceedings of the 6th Advanced International Conference on Telecommunications (AICT 2010), Barcelona, Spain, 9-15 May, 2010.

[7] V. Stavroulaki, et al., Opportunistic networks: an approach for exploiting cognitive radio networking technologies in the future Internet, IEEE Veh. Technol. Mag. 6 (3) (2011) 52-59.

[8] I. Comsa, M. Aydin, S. Zhang, P. Kuonen, J. Wagen, Reinforcement learning based radio resource scheduling in LTE-advanced, in: Proceedings of the 8th International Conference on Automation and Computing (ICAC), Karlsruhe, Germany, 1418 June, 2011.

[9] F. Bernardo, R. Agustí, J. Pérez-Romero, O. Sallent, An application of reinforcement learning for efficient spectrum usage in next-generation mobile cellular networks, IEEE Trans. Syst. Man Cybern. 40 (4) (2010) 477-484.

[10] Q. Zhao, L. Tong, A. Swami, Y. Chen, Decentralized cognitive MAC for opportunistic spectrum access in ad hoc networks: a POMDP framework, IEEE J. Sel. Areas Commun. 25 (3) (2007) 589-600.

[11] A. Raschellà, J. Pérez-Romero, O. Sallent, A. Umbert, On the use of POMDP for spectrum selection in cognitive radio networks, in: Proceedings of the 8th International Conference on Cognitive Radio Oriented Wireless Networks (CROWNCOM), Washington DC, United States, 8-10 July, 2013.
[12] A. Raschellà, J. Pérez-Romero, O. Sallent, A. Umbert, On the impact of the observation strategy in a POMDP-based framework for spectrum selection, in: Proceedings of the 24th Personal Indoor and Mobile Radio Communications (PIMRC), London, UK, 8-11 September, 2013.

[13] M. Matinmikko, T. Rauma, M. Mustonen, I. Harjula, H. Sarvanko, A. Mämmelä, Application of fuzzy logic to cognitive radio systems, IEICE Trans. B 92 (12)(2009) $3572-3580$.

[14] H. Ahmadi, I. Macaluso, L.A. DaSilva, Carrier Aggregation as a repeated game: learning algorithms for efficient convergence to a Nash equilibrium, in: Proceedings of the Global Communications Conference (GLOBECOM), Atlanta, USA, 9-13 December, 2013.

[15] G. Araniti, M. Condoluci, A. Iera, L. Militano, B.A. Pansera, Analytical modeling of bargaining solutions for multicast cellular services, Electron. J. Differ. Equ. 2013 (165) (2013) $1-10$

[16] ETSI, TC RRS, Reconfigurable Radio Systems (RRS); Functional Architecture (FA) for the Management and the Control of Reconfigurable Radio Systems, Technical Report 102682 V1.1.1, ETSI, July 2009.

[17] J. Gebert (Ed.) "OneFIT functional and system architecture", Deliverable D2.2 of OneFIT project, February 2011. Available at: http://www.ict-onefit.eu/ (accessed January 2015).

[18] F. Bouali, O. Sallent, J. Pérez-Romero, R. Agustí, A cognitive management framework for spectrum selection, Comput. Netw. J. 57 (14) (October 2013) 2752-2765.

[19] F. Bouali, O. Sallent, J. Pérez-Romero, R. Agustí, A framework based on a fittingness factor to enable efficient exploitation of spectrum opportunities in cognitive radio networks, in: Proceedings of the 14th International Symposium on Wireless Personal Multimedia Communications (WPMC), Brest, France, 3-6 October, 2011.

[20] A. Raschellà, A. Umbert, J. Pérez-Romero, O. Sallent, On demonstrating spectrum selection functionality for opportunistic networks, in: Proceedings of the 2nd International Conference on Mobile Services, Resources, and Users (MOBILITY 2012), Venice, Italy, 21-26 October, 2012.

[21] A. Raschellà, A. Umbert, J. Pérez-Romero, O. Sallent, A testbed platform to demonstrate spectrum selection in opportunistic networks, in: Proceedings of the 6th Joint IFIP Wireless and Mobile Networking Conference (WMNC 2013), Dubai, United Arab Emirates, 23-25 April, 2013.

[22] A. Raschellà, J. Pérez-Romero, O. Sallent, A. Umbert, Evaluation of a belief-based decision making in a real-time platform for cognitive radio networks, in: Proceedings of the 9th International Conference on Cognitive Radio Oriented Wireless Networks (CROWNCOM 2014), Oulu, Finland, 02-04 June, 2014.

[23] ]. Pérez-Romero, A. Raschellà, O. Sallent, A. Umbert, A belief-based decision making framework for spectrum selection in cognitive radio networks, IEEE Trans. Veh. Technol. (2015) in press.

[24] Sun Hongjian, A. Nallanathan, Wang Cheng-Xiang, Chen Yunfei, Wideband spectrum sensing for cognitive radio networks: a survey, IEEE Wirel. Commun. 20 2013) 74-81.

[25] Chunxiao Jiang, Yan Chen, Yang Gao, K.J. Ray Liu, Joint spectrum sensing and access evolutionary game in cognitive radio networks, IEEE Trans. Wirel. Commun, $12(5)(2013) 2470-2483$.

[26] Chunxiao Jiang, Yan Chen, K.J. Ray Liu, Multi-Channel sensing and access game: bayesian social learning with negative network externality, IEEE Trans. Wirel. Commun. 13 (4) (2014) 2176-2188.

[27] Federal Communications Commission (FCC), "ET Docket no. 03-287: Notice of proposed rule making and order", Federal Communications Commission (FCC), November 2003.

[28] C.F. Li James, Wei Zhang, Jinhong Yuan, Opportunistic spectrum sharing in cognitive radio networks based on primary limited feedback, IEEE Trans. Commun. 59 (12) (2011) 3272-3277.

[29] James C.F. Li, Wei Zhang, Aria Nosratinia, Jinhong Yuan, SHARP: Spectrum harvesting with ARQ retransmission and probing in cognitive radio, IEEE Trans. Commun. 61 (3) (2013) 951-960.

[30] Chunxiao Jiang, Yan Chen, K.J. Ray Liu, Yong Ren, Renewal-theoretical dynamic spectrum access in cognitive radio network with unknown primary behavior, IEEE J. Sel. Areas Commun. 31 (3) (2013) 406-416.

[31] D.R. Cox, Renewal Theory, Butler and Tanner, 1967.

[32] J. Vartiainen, M. Hoyhtya, J. Lehtomaki, T. Braysy, Priority channel selection based on detection history database, in: Proceedings of the 5th International Conference on Cognitive Radio Oriented Wireless Networks (CROWNCOM), Cannes, France, 9-11 June, 2010.

[33] Y. Li, Y. Dong, H. Zhang, H. Zhao, H. Shi, X. Zhao, QoS provisioning spectrum decision algorithm based on predictions in cognitive radio networks, in: Proceedings of the 6th International Conference on Wireless Communications, Networking and Mobile Computing (WiCOM), Chengdu, China, 23-25 September, 2010.

[34] W.-Y. Lee, I. Akyldiz, A spectrum decision framework for cognitive radio networks, IEEE Trans. Mob. Comput. 10 (2) (2011) 161-174.

[35] M. Höyhtyä, J. Vartiainen, H. Sarvanko, A. Mämmelä, Combination of short term and long term database for cognitive radio resource management, in: Proceedings of the 3rd International Symposium on Applied Sciences in Biomedical and Communication Technologies (ISABEL), Rome, Italy, 7-10 November, 2010.

[36] Chunxiao Jiang, Haijun Zhang, Yong Ren, Hsiao-Hwa Chen, Energy-efficient noncooperative cognitive radio networks: micro, meso and macro views, IEEE Commun. Mag. 52 (7) (July 2014) 14-20.

[37] S. Mubaraq Mishra, D. Cabric, C. Chang, D. Willkomm, B. van Schewick, A. Wolisz, R.W. Brodersen, A real time cognitive radio testbed for physical and link layer experiments, in: Proceedings of the Dynamic Spectrum Access Networks (DySPAN), Baltimore MD, USA, 8-11 November, 2005.

[38] P.D. Sutton, J. Lotze, H. Lahlou, S.A. Fahmy, K.E. Nolan, B. Ozgul, T.W. Rondeau, J. Noguera, L.E. Doyle, Iris: an architecture for cognitive radio networking testbeds, IEEE Commun. Mag. 48 (2010) 114-122. 
[39] Christian J. Rieser, Thomas W. Rondeau, Charles W. Bostian, Timothy M. Gallagher, Cognitive radio testbed: further details and testing of a distributed genetic algorithm based cognitive engine for programmable radios, in: Proceedings of Military Communications Conference (MILCOM), Monterey, CA, 1-3 November, 2004.

[40] T.R. Newman, A. He, J. Gaeddert, B. Hilburn, T. Bose, J.H. Reed, Virginia tech cognitive radio network testbed and open source cognitive radio framework, in: Proceedings of the 5th International Conference on Testbeds and Research Infrastructures for the Development of Networks \& Communities (TridentCom), Washington DC, United States, 6-8 April, 2009.

[41] D. Raychaudhuri, I. Seskar, M. Ott, S. Ganu, K. Ramachandran, H. Kremo, R. Siracusa, H. Liu, M. Singh, Overview of the ORBIT radio grid testbed for evaluation of next-generation wireless network protocols, in: Proceedings of the Wireless Communications and Networking Conference (WCNA), New Orleans LA, United States, 13-17 March, 2005.

[42] OneFIT Deliverable D4.1 "Formulation, implementation considerations, and first performance evaluation of algorithmic solutions", 2011.

[43] O. Moreno (Ed.) "Business scenarios, technical challenges and system requirements", Deliverable D2.1 of OneFIT ICT project, October 2010. Available at: http: //www.ict-onefit.eu/ (accessed January 2015).

[44] ICT EU (Opportunistic networks and Cognitive Management Systems for Efficient Application Provision in the Future InterneT) OneFIT Project. http://www. ict-onefit.eu/ (accessed January 2015).
[45] J. Pérez-Romero, A. Raschellà, O. Sallent, A. Umbert, Enhanced cognitive radio operation through belief-based decision making, in: Proceedings of the 20th European Wireless Conference (EW 2014), Barcelona, Spain, 1-16 May, 2014.

[46] L. Kleinrock, Queueing Systems. Volume I: Theory, John Wiley \& Sons, 1975.

[47] Ettus Research ${ }^{\mathrm{TM}}$ Distribution < http://www.ettus.com > (accessed March 2010).

[48] Free and Open Software Distribution < http://www.gnuradio.org > (accessed April 2010).

[49] J.J. Lehtomaki, M. Juntti, H. Saarnisaari, S. Koivu, Threshold setting strategies for a quantized total power radiometer, IEEE Signal Processing Letters 12 (11) (November 2005) 796-799.

[50] M. López-Benítez, F. Casadevall, Methodological aspects of spectrum occupancy evaluation in the context of cognitive radio, in: Proceedings of the European Transactions on Telecommunications (Wiley), Special Issue on European Wireless 2009 Conference, 1, December 2010, pp. 680-693.

[51] T. Wierzbowski (Ed.) "Protocols, performance assessment and consolidation on interfaces for standardization", Deliverable D3.3 of OneFIT ICT project, June 2012

[52] ECMA, MAC and PHY for operation in TV white space, in: European association for standardizing information and communication systems, Tech. Rep. ECMA-392, December 2009. 